\title{
Optimal Consumption and Investment Strategies with Stochastic Interest Rates
}

\author{
Munk, Claus; Sørensen, Carsten
}

Document Version

Final published version

Publication date:

2000

License

CC BY-NC-ND

Citation for published version (APA):

Munk, C., \& Sørensen, C. (2000). Optimal Consumption and Investment Strategies with Stochastic Interest Rates. Institut for Finansiering, Copenhagen Business School. Working Papers / Department of Finance.

Copenhagen Business School No. 2000-9

Link to publication in CBS Research Portal

\footnotetext{
General rights

Copyright and moral rights for the publications made accessible in the public portal are retained by the authors and/or other copyright owners and it is a condition of accessing publications that users recognise and abide by the legal requirements associated with these rights.

Take down policy

If you believe that this document breaches copyright please contact us (research.lib@cbs.dk) providing details, and we will remove access to the work immediately and investigate your claim.
} 
WP 2000-9

Optimal Consumption and Investment Strategies with Stochastic Interest Rates

af

Claus Munk \& Carsten Sørensen

INSTITUT FOR FINANSIERING, Handelshøjskolen i København

Solbjerg Plads 3, 2000 Frederiksberg C

tlf.: 38153615 fax: 38153600

DEPARTMENT OF FINANCE, Copenhagen Business School

Solbjerg Plads 3, DK - 2000 Frederiksberg C, Denmark

Phone (+45)38153615, Fax (+45)38153600

www.cbs.dk/departments/finance

ISBN 87-90705-43-2

ISSN 0903-0352 


\section{Optimal Consumption and Investment Strategies with Stochastic Interest Rates*}

\author{
Claus Munk \\ Dept. of Accounting, Finance \& Law \\ SDU - Odense University \\ Campusvej 55 \\ DK-5230 Odense M \\ Denmark
}

\author{
Carsten Sørensen \\ Department of Finance \\ Copenhagen Business School \\ Solbjerg Plads 3 \\ DK-2000 Frederiksberg \\ Denmark
}

* We thank for comments and suggestions from seminar participants at Copenhagen Business School. Both authors gratefully acknowledge financial support from the Danish Research Council for Social Sciences. 


\title{
Optimal Consumption and Investment Strategies with Stochastic Interest Rates
}

\begin{abstract}
We study the consumption and investment choice of a time-additive power utility investor and demonstrate how the investor should optimally hedge changes in the opportunity set. The investor is allowed to invest in stocks and interest rate dependent assets in a continuous-time dynamically complete market. In particular, we demonstrate that under stochastic interest rates the investor optimally hedges changes in the term structure of interest rates by investing in a coupon bond, or portfolio of bonds, with a payment schedule that matches the forward-expected (i.e certainty equivalent) consumption pattern. This is of conceptual importance since the hedge portfolio does not depend on the specific term structure dynamics (only through the consequences for the optimal consumption pattern). We consider two explicit examples where the dynamics of the term structure of interest rates are given by the Vasicek-model and a three-factor non-Markovian Heath-Jarrow-Morton model.
\end{abstract}




\section{Introduction}

In this paper, we establish specific results on how investors in a continuous-time model should optimally hedge changes in the investment opportunity set, in the sense of Merton $(1971,1973)$. In particular, under specialized (Gaussian) term structure dynamics and dynamically complete markets, we demonstrate that the optimal way to hedge changes in the term structure of interest rates is by investing in a coupon bond, or portfolio of bonds, with a payment schedule that matches the forward-expected (i.e certainty equivalent) consumption pattern. This provides a conceptual way to think about how to hedge changes in the opportunity set where the focus is on the optimal consumption pattern and not the assumed dynamics of the opportunity set.

We consider the optimal consumption and investment problem of an investor with timeadditive power utility, i.e. with a Constant Relative Risk Aversion (CCRA) utility function. Throughout the paper we allow for non-Markovian dynamics of asset prices and the term structure of interest rates. In particular, under specialized Gaussian term structure dynamics we use the framework of Heath, Jarrow and Morton (1992) (HJM) which as input uses the current form of the term structure of interest rates. This allows us to address questions like: Is the current form of the term structure important for how to hedge changes in the opportunity set or does only the dynamics of the term structure matter? We address this question by considering two explicit examples based on the Vasicek (1977) model as well as a HJM three-factor model where the term structure can exhibit three kinds of changes: a parallel shift, a slope change, and a curvature change. Our results suggest that the form of the initial term structure of interest rates is of crucial importance for the (forward-expected) consumption pattern and, hence, important for the relevant bond to hedge changes in the opportunity set while the specific dynamics of the term structure is of minor importance.

Our specific results and examples also provide a contribution on its own since these are, to our knowledge, the first explicit results to an intertemporal consumption and investment problem where the dynamics of the opportunity set is non-Markovian and the investor has non-logarithmic utility.

The intertemporal consumption and investment decision of a utility-maximizing investor is a classical problem of financial economics. In two pathbreaking papers, Merton $(1969,1971)$ studies this problem in a continuous-time framework using dynamic programming. He shows that the optimal investment strategy combines a myopic, or speculative, portfolio and some portfolio which hedges changes in the investment opportunity set (i.e. time-varying returns and volatilities). Also, Merton (1973) addresses the optimization problem under a stochastic investment opportunity set where the drift and diffusion coefficients of the asset prices depend 
on the current short-term interest rate, but he does not derive explicit results for the hedge portfolio in this case.

Our paper deviates from the classical Merton $(1969,1971,1973)$ papers in at least three methodological aspects: (i) We do not assume that asset prices are Markovian, (ii) we assume complete markets, and (iii) instead of using a dynamic programming approach we use the martingale solution approach suggested and formalized by Cox and Huang $(1989,1991)$ and Karatzas, Lehoczky, and Shreve (1987).

In the literature there has recently been a number of studies of optimal investment strategies in dynamic markets with specific assumptions on the changes in the investment opportunity set. Brennan, Schwartz and Lagnado (1997) solve numerically for the optimal investment strategy of an investor with CRRA utility from terminal wealth in a market with stocks and bonds driven by three variables: the short-term and the long-term interest rates and a stochastic dividend yield on the stock. They investigate numerically how the optimal investment strategy deviates from the myopic strategy for different investment horizons.

Brennan and Xia (1998) and Sørensen (1999) consider the investment problem of a CRRA utility investor with utility from terminal wealth only. They assume complete markets and show that in the case where the term structure of interest rates is described by a Vasicek-model and market prices on risk (and excess returns) are constant, the optimal hedge portfolio is the zerocoupon bond that expires at the investment horizon. Also, Liu (1999) provide a similar result in an example and this particular result is also a very special case of the results obtained in this paper.

Campbell and Viceira (1998) consider a dynamic investment problem that allows for intertemporal consumption. In fact, their (recursive) preferences are more general than the CRRA utility assumed in this paper. On the other hand we allow for much more general dynamics of the opportunity set. While Campbell and Viceira (1998) provide explicit (but approximate) results on how to hedge changes in interest rates, they do not explicitly link the optimal hedge portfolio to the optimal consumption pattern.

Detemple, Garcia and Rindisbacher (1999) consider a complete market where changes in the investment opportunity set are driven by a multi-dimensional state variable following a Markov diffusion. For an investor maximizing utility of terminal wealth, they are able to express the optimal investment strategy as a combination of the myopic, growth-optimal strategy and two terms representing the hedge against changes in the short-term interest rate and the market prices of risk, respectively. The two hedge terms involves Malliavin derivatives and are based on the Clark-Ocone formula, cf. Ocone and Karatzas (1991). In the special setting of a CRRA 
investor in a complete market with a single risky asset where the short-term interest rate and the market price of risk follow two particular diffusion processes that are both perfectly correlated with the risky asset price, they are able to derive the hedge strategies in closed-form. For other settings they demonstrate how to compute the hedge components numerically by simulations using their Malliavin formulation. Again, they do not explicitly link the optimal hedge portfolio to the optimal consumption pattern, as in this paper.

Kim and Omberg (1996), Campbell and Viceira (1999), Chacko and Viceira (1999), and other recent papers consider dynamic optimization problems where interest rates are constant but where risk premia are stochastic and/or markets are incomplete; see also Campbell (2000) for a survey of the literature.

In contrast to all the papers referred above, we allow for non-Markovian dynamics of the opportunity set and link the optimal hedge portfolio to the optimal consumption pattern of the investor.

The rest of the paper is organized as follows. In Section 2 we set up the general continuoustime consumption and investment problem in a dynamically complete market and provide a general characterization of the optimal consumption and investment policy for a CRRA investor in a possibly non-Markovian setting. In Section 3 we derive explicit results on how to hedge changes in the term structure of interest rates using coupon bonds in a specialized HJM multifactor Gaussian term structure setting. In Section 4 we consider two specific numerical examples based on the Vasicek-model and an HJM three-factor model. Section 5 concludes and proofs are given in an Appendix.

\section{The investment problem}

We consider a frictionless economy where the dynamics are generated by a $d$-dimensional Wiener process, $w=\left(w_{1}, \ldots, w_{d}\right)$, defined on a probability space $(\Omega, \mathcal{F}, \mathbb{P}) . F=\left\{\mathcal{F}_{t}: t \geq 0\right\}$ denotes the standard filtration of $w$ and, formally, $(\Omega, \mathcal{F}, F, \mathbb{P})$ is the basic model for uncertainty and information arrival in the following.

\section{$2.1 \quad$ Preferences}

We will consider the investment problem of an expected utility maximizing investor with a time-separable constant relative risk aversion utility function on the form,

$$
K \cdot \mathrm{E}_{0}\left[\int_{0}^{T} U_{1}\left(C_{t}, t\right) d t\right]+(1-K) \cdot \mathrm{E}_{0}\left[U_{2}\left(W_{T}\right)\right]
$$


where

$$
U_{1}(C, t)=e^{-\beta t} \frac{C^{1-\gamma}-1}{1-\gamma} \text { and } \quad U_{2}(W)=e^{-\beta T} \frac{W^{1-\gamma}-1}{1-\gamma}
$$

and where $\beta$ is a constant subjective time discount rate and $\gamma$ is a constant relative risk aversion parameter. The preference parameter $K$ controls the relative weight of intermediate consumption, $C_{t}$, and terminal wealth, $W_{T}$, in the agent's utility function. The special case where $\gamma=$ 1 is the logarithmic utility case: $U_{1}(C, t)=e^{-\beta t} \log C$ and $U_{2}(W)=e^{-\beta T} \log W$.

\section{$2.2 \quad$ Investment assets}

The agent can invest in a set of financial securities. One of these financial assets is assumed to be an "instantaneously" risk-free bank account which has a return equal to the short-term interest rate $r_{t}$. In addition, the agent can invest in $d$ risky assets with prices described by the vector $V_{t}=\left(V_{1 t}, \ldots, V_{d t}\right)^{\prime}$. The price dynamics of the risky assets (cum dividend) are given by

$$
d V_{t}=\operatorname{diag}\left(V_{t}\right)\left[\left(r_{t} 1_{d}+\sigma_{t} \lambda_{t}\right) d t+\sigma_{t} d w_{t}\right]
$$

where $\lambda_{t}$ is an $\mathbb{R}^{d}$-valued stochastic process of market prices of risk, $\sigma_{t}$ is an $\mathbb{R}^{d \times d}$-valued stochastic process of volatilities, $1_{d}$ is a $d$-dimensional vector of ones, and $\operatorname{diag}\left(V_{t}\right)$ is a $(d \times d)$ dimensional matrix with $V_{t}$ in the diagonal (and zeros off the diagonal). It is assumed that $\sigma$ has full rank $d$ implying that markets are dynamically complete, c.f. Duffie and Huang (1985). As a consequence of markets being dynamically complete, the pricing kernel (or state-price deflator) is uniquely determined and given by (see, e.g., Duffie (1996), chapter 6)

$$
\zeta_{t}=\exp \left\{-\int_{0}^{t} r_{s} d s-\int_{0}^{t} \lambda_{s}^{\prime} d w_{s}-\frac{1}{2} \int_{0}^{t}\left\|\lambda_{s}\right\|^{2} d s\right\}, t \geq 0
$$

or, equivalently, in differential form,

$$
d \zeta_{t}=\zeta_{t}\left[-r_{t} d t-\lambda_{t} d w_{t}\right], \zeta_{0}=1
$$

The present value of any stochastic payoff, $X$, paid at some future time point $s$ can be determined by evaluating the pricing kernel weighted payoff. In particular, we have

$$
\mathrm{PV}_{t}[X]=\mathrm{E}_{t}\left[\left(\frac{\zeta_{s}}{\zeta_{t}}\right) X\right]=P_{t}(s) \hat{\mathrm{E}}_{t}^{s}[X]
$$

where $P_{t}(s)$ is the time $t$ price on a zero-coupon bond that expires at time $s$. The last equality defines the so-called certainty-equivalent or forward-expected payoff, $\hat{\mathrm{E}}_{t}^{s}[X]$; see e.g. Jamshidian $(1987,1989)$ and Geman $(1989)$ who introduce the notion of the forward-expected martingale measure, as being distinct from the usual risk-neutral martingale measure in the context of interest rate models. 


\subsection{The problem and the general solution}

Let $\pi_{t}$ be an $\mathbb{R}^{d}$-valued process that describes the fractions of wealth that the agent allocates into the $d$ different risky assets. The wealth of the agent then evolves according to

$$
d W_{t}=\left[\left(r_{t}+\pi_{t}^{\prime} \sigma_{t} \lambda_{t}\right) W_{t}-C_{t}\right] d t+W_{t} \pi_{t}^{\prime} \sigma_{t} d w_{t}
$$

The agent's problem is to choose a dynamic consumption strategy, $C_{t}$, and portfolio policy, $\pi_{t}$, in order to maximize the expected utility in (1). The main idea of the martingale solution approach suggested and formalized by Cox and Huang $(1989,1991)$ and Karatzas, Lehoczky, and Shreve (1987) is to alternatively consider the static problem

$$
\sup _{\left\{C_{t}, W_{T}\right\}} K \cdot \mathrm{E}_{0}\left[\int_{0}^{T} U_{1}\left(C_{t}, t\right) d t\right]+(1-K) \cdot \mathrm{E}_{0}\left[U_{2}\left(W_{T}\right)\right]
$$

subject to

$$
\mathrm{E}_{0}\left[\int_{0}^{T}\left(\frac{\zeta_{t}}{\zeta_{0}}\right) C_{t} d t+\left(\frac{\zeta_{T}}{\zeta_{0}}\right) W_{T}\right] \leq W_{0} .
$$

The problem in (7) and (8) is a standard Lagrangian optimization problem which can be solved using the Saddle Point Theorem (see e.g., Duffie (1996), pp. 205-208) to determine the optimal consumption process, $C_{t}$, and terminal wealth, $W_{T}$. In principle, the problem is to maximize expected utility subject to the budget constraint (8) which states that the present value of the consumption stream and terminal wealth cannot exceed the agent's current wealth. The value function, or indirect utility, $J_{t}$ from the optimization problem is the maximum expected remaining life-time utility which can be achieved by the optimal consumption and terminal wealth plan following any time point $t, 0 \leq t \leq T$.

As shown by Cox and Huang $(1989,1991)$ and Karatzas, Lehoczky, and Shreve (1987), the solution to this problem also provides the solution to the dynamic problem of choosing the optimal consumption strategy and portfolio policy. While the consumption policy is given explicitly when solving (7) and (8), the optimal portfolio policy is only given implicitly as the policy which replicates the optimal terminal wealth from the above problem and in accordance with (6).

As described by Merton $(1971,1973)$, the optimal investment policy can in a general Markovian setting be decomposed into a speculative portfolio (chosen by a myopic or logarithmic utility investor) and a term which hedges changes in the opportunity set. For general utility functions, the optimal investment strategy can be represented rather abstractly in complete markets in terms of stochastic integrals of Malliavin derivatives by the Clark-Ocone formula, cf. Ocone and Karatzas (1991). However, in order to derive an explicit expression for the optimal portfolio 
for non-logarithmic utility functions it is generally recognized that the price dynamics must be specialized. Cox and Huang (1989) show that when the state-price deflator and the risky asset prices constitute a Markovian system, the optimal investment strategy can be represented in terms of the solution of a linear second order partial differential equation. On the other hand, in the following proposition we provide a closed-form expression for the optimal investment strategy for a power utility investor in a general non-Markovian complete market setting for a CRRA investor. The (investor specific) stochastic process

$$
Q_{t}=K^{\frac{1}{\gamma}} \int_{t}^{T} e^{-\frac{\beta}{\gamma}(s-t)} \mathrm{E}_{t}\left[\left(\frac{\zeta_{s}}{\zeta_{t}}\right)^{\frac{\gamma-1}{\gamma}}\right] d s+(1-K)^{\frac{1}{\gamma}} e^{-\frac{\beta}{\gamma}(T-t)} \mathrm{E}_{t}\left[\left(\frac{\zeta_{T}}{\zeta_{t}}\right)^{\frac{\gamma-1}{\gamma}}\right]
$$

is crucial for how to hedge changes in the opportunity set, as will be formalized in the proposition below.

Since $Q_{t}$ is a positive stochastic process adapted to the filtration generated by $w_{t}$, it follows from the Martingale Representation Theorem (see e.g., Duffie (1996)), that the dynamics of $Q_{t}$ can be described on the form

$$
d Q_{t}=Q_{t}\left[\mu_{Q t} d t+\sigma_{Q t} d w_{t}\right]
$$

for some drift process $\mu_{Q t}$ and some volatility process $\sigma_{Q t}$.

Proposition 1 The value function of the general problem in (7) and (8) has the form

$$
J_{t}=\frac{Q_{t}^{\gamma} W_{t}^{1-\gamma}-A(t)}{1-\gamma}
$$

where

$$
A(t)=\frac{K}{\beta}\left(1-e^{-\beta(T-t)}\right)+(1-K) e^{-\beta(T-t)}
$$

and $Q_{t}$ is defined in equation (9).

The optimal consumption choice and the optimal portfolio policy at time $t$ are given by

$$
C_{t}=K^{\frac{1}{\gamma}} \frac{W_{t}}{Q_{t}}
$$

and

$$
\pi_{t}=\left(\frac{1}{\gamma}\right)\left(\sigma_{t}^{\prime}\right)^{-1} \lambda_{t}+\left(\sigma_{t}^{\prime}\right)^{-1} \sigma_{Q t}
$$

Proof: See the appendix.

Proposition 1 states the optimal portfolio policy has the same form as in Merton (1971). The portfolio policy can be decomposed into a speculative portfolio (the first term in (13)) and a hedge portfolio that describes how the investor should optimally hedge changes in the 
investment opportunity set (the last term in (13)). As usual the hedge portfolio vanishes in the special case where the investor has logarithmic utility; the precise result for the logarithmic investor benchmark case is stated explicitly in a corollary below. Also, note that the optimal consumption choice in (12) is on the same "feed-back form" as in, e.g., Merton (1971) even though Proposition 1 holds for a general non-Markovian setting where dynamic programming is not directly applicable.

It is seen from Proposition 1 that in order to hedge changes in the opportunity set, the investor must form a hedge portfolio that basically mimics the dynamics of $Q_{t}$. Hence, $Q_{t}$ reflects everything of importance for how to hedge changes in the investment opportunity set. For a given investor it can thus be inferred from (9) that only processes included in the description of (moments of) the pricing kernel stated in (3) are relevant for intertemporal hedging purposes. In general, the investor should alone consider to hedge changes in interest rates and changes in prices on risk in the economy while changes in, say, volatilities on marketed securities should be of no concern in our complete market setting.

In order to discuss the implications of the results in Proposition 1 we will focus on two benchmark cases: the log-utility case $(\gamma=1)$ and the case of an infinitely risk averse investor $(\gamma$ $=\infty) .{ }^{1}$ These two investor types represent important polar cases since the logarithmic investor does not hedge changes in the opportunity set at all while the infinitely risk averse investor has no speculative demand for securities at all. Moreover, in the next section we will demonstrate that the forward expected consumption pattern of the agent is important for how to hedge changes in the term structure of interest rates in a specialized market setting. As formalized and explicitly stated in the following corollaries, the forward-expected consumption patterns of the benchmark cases of log-utility investors and infinitely risk averse investors do not depend on the dynamics in the investment opportunity set but only on the current form of the term structure of interest rates.

Corollary 1 For the special case of an investor with logarithmic utility, i.e. $\gamma=1$, the value function general problem in (7) and (8) has the form

$$
J_{t}=Q_{t} \log W_{t}
$$

with $Q_{t}=A(t)$ and where $A(t)$ is defined in (11).

The optimal consumption choice and the optimal portfolio policy at time $t$ are given by

$$
C_{t}=K \frac{W_{t}}{Q_{t}}
$$

\footnotetext{
${ }^{1}$ Formally, the results for an infinitely risk averse investor are defined as the limiting results as $\gamma \rightarrow \infty$.
} 
and

$$
\pi_{t}=\left(\sigma_{t}^{\prime}\right)^{-1} \lambda_{t}
$$

Furthermore, at time $t$ the forward-expected consumption rates at future time points $s \in[t, T)$ are given by

$$
\hat{\mathrm{E}}_{t}^{s}\left[C_{s}\right]=C_{t}\left(P_{t}(s)\right)^{-1} e^{-\beta(s-t)}, 0 \leq t \leq s<T,
$$

and the forward-expected terminal wealth at time $T$ is given by

$$
\hat{\mathrm{E}}_{t}^{T}\left[W_{T}\right]=C_{t}\left(\frac{1-K}{K}\right)\left(P_{t}(T)\right)^{-1} e^{-\beta(T-t)} .
$$

Proof: See the appendix.

Corollary 2 For the special case of an infinitely risk averse investor, i.e. $\gamma=\infty$, the optimal consumption choice is constant and given by

$$
C_{t}=\frac{W_{t}}{Q_{t}}=\frac{W_{0}}{Q_{0}}
$$

and the optimal portfolio policy at time $t$ is given by

$$
\pi_{t}=\left(\sigma_{t}^{\prime}\right)^{-1} \sigma_{Q t}
$$

with

$$
Q_{t}=\int_{t}^{T} P_{t}(s) d s+P_{t}(T)
$$

In particular, the forward-expected consumption rates at future time points $s \in[t, T)$ are given by

$$
\hat{\mathrm{E}}_{t}^{s}\left[C_{s}\right]=C_{t}, 0 \leq t \leq s<T,
$$

and the forward-expected terminal wealth at time $T$ is given by

$$
\hat{\mathrm{E}}_{t}^{T}\left[W_{T}\right]=C_{t}, 0 \leq t \leq s<T .
$$

Proof: See the appendix.

Corollary 1 states that the optimal portfolio choice of a logarithmic utility investor is described entirely by the speculative portfolio while Corollary 2 states that the optimal portfolio choice of an infinitely risk averse investor is described entirely by the hedge portfolio. The hedge portfolio, as reflected in $Q_{t}$, will in this case be an annuity bond ${ }^{2}$ and the optimal consumption will be certain and "flat" and basically be generated by the certain payments on the annuity bond. ${ }^{3}$

\footnotetext{
${ }^{2}$ An annuity bond is a coupon bond where the certain cash flows (coupon + principal repayment) from the bond are the same throughout the finite life of the bond (and including the last payment on the bond).

${ }^{3}$ Wachter (1999) provides similar results for an infinitely risk averse investors in a Markovian market setting.
} 


\section{$3 \quad$ Hedging changes in interest rates}

In the following, we are especially interested in how the agent should allocate investment funds into two general classes of securities: stocks and bonds. Therefore, we will introduce additional notation and separate the investment assets into stocks and bonds. Formally, we will split the $d$-dimensional Wiener process generating the financial asset returns as $w=\left(w_{B}, w_{S}\right)$, where $w_{B}$ is of dimension $k$ and $w_{S}$ of dimension $l=d-k$. We assume that the dynamics of the term structure of interest rates, and, hence, the dynamics of prices on bonds and other term structure derivatives traded at the bond market, are affected only by $w_{B}$. The dynamics of the prices of the stocks may depend on both $w_{B}$ and $w_{S}$ which allows for correlation between stocks and term structure derivatives. Specifically, the investor can invest in the "instantaneously" risk-free bank account, $k$ term-structure derivatives, and $l$ stocks. The asset price dynamics are given by

$$
d B_{t}=\operatorname{diag}\left(B_{t}\right)\left[\left(r_{t} 1_{k}+\sigma_{B t} \lambda_{B t}\right) d t+\sigma_{B t} d w_{B t}\right]
$$

and

$$
d S_{t}=\operatorname{diag}\left(S_{t}\right)\left[\left(r_{t} 1_{l}+\varphi_{S t}\right) d t+\sigma_{S 1 t} d w_{B t}+\sigma_{S 2 t} d w_{S t}\right]
$$

where $\sigma_{B}, \sigma_{S 1}$, and $\sigma_{S 2}$ are matrix valued processes of dimension $k \times k, l \times k$, and $l \times l$, respectively. Again, $\sigma_{B}$ and $\sigma_{S 2}$ are assumed non-singular so that markets are complete. Changes in the returns of the term structure derivatives and the stocks are correlated with $k \times l$ covariance matrix $\sigma_{B t} \sigma_{S 1 t}^{\prime}$. The market price of risk process $\lambda$ (which is not dependent on the particular set of assets chosen) has the form

$$
\lambda_{t}=\left(\lambda_{B t}, \lambda_{S t}\right)^{\prime}
$$

where

$$
\lambda_{S t}=\sigma_{S 2 t}^{-1} \varphi_{S t}-\sigma_{S 2 t}^{-1} \sigma_{S 1 t} \lambda_{B t} .
$$

Note that we have introduced the $\mathbb{R}^{l}$-valued stochastic process $\varphi_{S t}\left(=\sigma_{S 1 t} \lambda_{B t}+\sigma_{S 2 t} \lambda_{S t}\right)$ which can be interpreted as the expected excess return on the stocks.

\subsection{Term structure dynamics and portfolio choice}

In the following we will introduce additional notation in order to be specific about the term structure dynamics in the economy and in order to provide a framework for casting the specific examples considered in a later section. Specifically, we assume that the dynamics of the term structure of interest rates can be described by a $k$-factor model of the HJM-class introduced by Heath, Jarrow, and Morton (1992). Moreover, we will assume that forward rate volatilities are 
deterministic which implies that we only consider term structure dynamics within the so-called Gaussian HJM-class.

For any maturity date $\tau$ the dynamics of the $\tau$-maturity instantaneous forward rate are

$$
f_{t}(\tau)=f_{0}(\tau)+\int_{0}^{t} \alpha(s, \tau) d s+\int_{0}^{t} \sigma_{f}(s, \tau)^{\prime} d w_{B s}
$$

where $\sigma_{f}(\cdot, \tau)$ is an $\mathbb{R}^{k}$-valued deterministic function and $f_{0}(\tau)$ is the $\tau$-maturity forward rate observed initially at time 0 . The most important feature of the HJM-modeling is that in the absence of arbitrage, one only has to specify the initial term structure of forward rates and the volatility structure $\sigma_{f}(t, \tau)$ for all $t$ and $\tau$ in order to have a well-specified term structure model; in particular, as a no-arbitrage drift restriction, we have that

$$
\alpha(t, \tau)=\sigma_{f}(t, \tau)^{\prime}\left(\lambda_{B}(t)+\int_{t}^{\tau} \sigma_{f}(t, u) d u\right) .
$$

While equation (26) (and the no-arbitrage drift restriction) describes the evolvement over time of the entire forward rate curve, the dynamics of the short-term interest rate is given as the special case where $\tau=t$, i.e. $r_{t}=f_{t}(t)$. The dynamics of the short-term interest rate are thus described by

$$
r_{t}=f_{0}(t)+\int_{0}^{t} \alpha(s, t) d s+\int_{0}^{t} \sigma_{f}(s, t)^{\prime} d w_{B s} .
$$

Among the many term-structure derivatives, we focus on default-free bonds. The dynamics of the price $P_{t}(\tau)=\exp \left(-\int_{t}^{\tau} f_{t}(s) d s\right)$ of the zero-coupon bond maturing at time $\tau$ is given by

$$
d P_{t}(\tau)=P_{t}(\tau)\left[\left(r_{t}+\sigma_{P}(t, \tau)^{\prime} \lambda_{B}(t)\right) d t+\sigma_{P}(t, \tau)^{\prime} d w_{B t}\right]
$$

where $\sigma_{P}(t, \tau)=-\int_{t}^{\tau} \sigma_{f}(t, u) d u$. For later use we will also consider a bond paying a continuous coupon of $k(t)$ up to time $T$ and a lump sum payment of $k(T)$ at time $T$. The time $t$ price of such a bond is

$$
B_{t}^{\mathrm{cpn}}=\int_{t}^{T} k(s) P_{t}(s) d s+k(T) P_{t}(T) .
$$

Applying the Leibnitz-type rule for stochastic processes stated in Lemma 1 in the Appendix, we see that the coupon bond price evolves according to

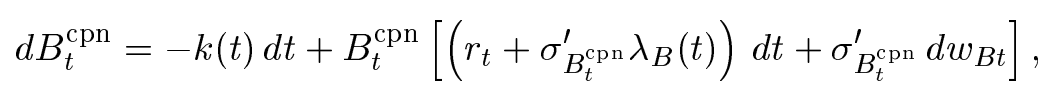

where

$$
\sigma_{B_{t}^{\mathrm{cpn}}}=\frac{\int_{t}^{T} k(s) P_{t}(s) \sigma_{P}(t, s) d s+k(T) P_{t}(T) \sigma_{P}(t, T)}{\int_{t}^{T} k(s) P_{t}(s) d s+k(T) P_{t}(T)} .
$$

Our specific results on how to hedge changes in interest rates, as stated in the following Proposition 2, are based on the following assumption. 
Assumption 1 The relative risk process $\lambda_{t} \equiv \lambda(t)$ and the forward rate volatilities $\sigma_{f}(t, \tau)$ are deterministic functions of time.

The implications of the assumption that prices of risk and forward rate volatilities are deterministic are important since we only allow interest rates to change and, hence, there are no reasons to hedge changes in prices of risk nor forward rate volatilities. Also, as a consequence of Assumption 1 the following analysis is limited to Gaussian models of the term structure of interest rates. However, note that we do not assume that the diffusion coefficients $\sigma_{B}, \sigma_{S 1}$, and $\sigma_{S 2}$ of the investment assets are deterministic and, in fact, they may be described by non-Markovian processes.

Despite shortcomings of Gaussian term structure models, such as not ruling out negative interest rates, multi-factor Gaussian models are often used for derivative pricing since they allow closed-form solution for most European-type term structure contingent claims, cf., e.g., Amin and Jarrow (1992) and Brace and Musiela (1994). As we shall see in the following, the Gaussian assumption also allows closed-form expressions for optimal investment strategies. Furthermore, it is important to point out that not even in Gaussian HJM-models is the short rate process necessarily Markovian. Only if $\sigma_{f}(t, \tau)$ can be separated as $\sigma_{f}(t, \tau)=G(t) H(\tau)$, where $H$ is a real-valued continuously differentiable function that never changes sign and $G$ is an $\mathbb{R}^{k}$-valued continuously differentiable function, is the short rate Markovian, cf. Carverhill (1994). This will e.g. not be the case in the HJM three-factor example considered in a subsequent section and, hence, the short-term interest rate will not be a Markovian process in the specific example.

From the assumption that prices of risk and forward rate volatilities are deterministic, it follows that the short-term interest rate in (27) is normally distributed (Gaussian) and that the pricing kernel $\zeta_{t}$, as stated in (3), is lognormally distributed. It is thus possible to compute in closed-form the expectations in the definition of $Q_{t}$ in (9) and, hence, obtain an analytical expression for $Q_{t}$. The proof of the following proposition is based on this feature.

Proposition 2 Assuming that the relative risk process $\lambda(t)$ and the forward rate volatilities $\sigma_{f}(t, \tau)$ are deterministic functions of time, the value function and the optimal consumption strategy are given by (11) and (12) in Proposition 1 where in this case

$$
Q_{t}=\int_{t}^{T} Z_{t}(s) d s+Z_{t}(T)
$$

with

$$
\begin{aligned}
& Z_{t}(s)=K^{\frac{1}{\gamma}}\left(P_{t}(s)\right)^{\frac{\gamma-1}{\gamma}} \exp \left\{-\frac{\beta}{\gamma}(s-t)+\frac{1-\gamma}{2 \gamma^{2}} g(t, s)\right\}, 0 \leq t \leq s<T \\
& Z_{t}(T)=(1-K)^{\frac{1}{\gamma}}\left(P_{t}(T)\right)^{\frac{\gamma-1}{\gamma}} \exp \left\{-\frac{\beta}{\gamma}(T-t)+\frac{1-\gamma}{2 \gamma^{2}} g(t, T)\right\}
\end{aligned}
$$


and

$$
g(t, s)=\int_{t}^{s}\|\lambda(u)\|^{2} d u+\int_{t}^{s}\left\|\sigma_{P}(u, s)\right\|^{2} d u-2 \int_{t}^{s} \lambda_{B}(u)^{\prime} \sigma_{P}(u, s) d u .
$$

The optimal portfolio policy at time $t$ is described by

$$
\pi_{t}=\left(\frac{1}{\gamma}\right) \sigma_{t}^{-1} \lambda(t)+\left(\frac{\gamma-1}{\gamma}\right) \sigma_{t}^{-1}\left(\begin{array}{c}
\sigma_{B_{t}^{\mathrm{cpn}}} \\
0
\end{array}\right)
$$

where $\sigma_{B_{t}^{\mathrm{cpn}}}$ is the volatility vector of a bond, as defined in equation (29), which pays continuous coupon according to

$$
k(s)=\hat{\mathrm{E}}_{t}^{s}\left[C_{s}\right]=K^{\frac{1}{\gamma}} \frac{W_{t}}{Q_{t}}\left(P_{t}(s)\right)^{-\frac{1}{\gamma}} \exp \left\{-\frac{\beta}{\gamma}(s-t)+\frac{1-\gamma}{2 \gamma^{2}} g(t, s)\right\}, 0 \leq t \leq s<T,
$$

and has a terminal lump sum payment at time $T$ of

$$
k(T)=\hat{\mathrm{E}}_{t}^{T}\left[W_{T}\right]=(1-K)^{\frac{1}{\gamma}} \frac{W_{t}}{Q_{t}}\left(P_{t}(T)\right)^{-\frac{1}{\gamma}} \exp \left\{-\frac{\beta}{\gamma}(T-t)+\frac{1-\gamma}{2 \gamma^{2}} g(t, T)\right\}
$$

Proof: See the appendix.

Proposition 2 states that in order to hedge changes in the opportunity set, i.e. changes in the term structure of interest rates in the present context, the relevant hedge portfolio is a coupon bond with coupons chosen to match the (forward-expected) consumption pattern. Specifically, Proposition 2 states that the optimal investment strategy allocates a fraction of wealth $(1 / \gamma)$ into the speculative portfolio and a fraction of wealth $(1-1 / \gamma)$ into the specific coupon bond. Since the portfolio weights in $\pi_{t}$ only describe the portfolio weights on the individual risky assets in the economy, the individual components in $\pi_{t}$ are not restricted to sum to one, and any excess wealth will be invested in the bank account. Furthermore, it follows directly that in the special case of investors with logarithmic utility $(\gamma=1)$ there are no desire for hedging changes in the opportunity set while on the other hand for very risk averse investors (i.e. as $\gamma \rightarrow \infty$ ) there are no speculative demand for risky assets, in accordance with Corollary 1 and Corollary 2.

The special case $K=0$ corresponds to utility from terminal wealth only and in this case it follows from Proposition 2 that the relevant bond for hedging changes in the term structure of interest rates is a bond that only has a lump sum payment at time $T$. In this special case Proposition 2 generalizes the insights of Brennan and Xia (1998) and Sørensen (1999) who in Vasicek settings demonstrate that, in the case of utility from terminal wealth only, the relevant bond for hedging changes in the opportunity set is the zero-coupon bond that expires at the investment horizon.

In our view the hedging strategy in Proposition 2 is of conceptual importance since the optimal way to hedge changes in the opportunity set is model-independent in the sense that 
the investor should alone aim at buying a coupon bond, or portfolio of bonds, such that the (forward-expected) consumption pattern is matched. In general, Proposition 2 states that the specific dynamics of the term structure of interest rates is of importance for how to hedge changes in the opportunity set only through its effect on the optimal (forward-expected) consumption pattern. In the following examples, we will focus on the determinants of the optimal (forwardexpected) consumption patterns and, in particular, we will focus on whether the current form of the term structure or the dynamics of the term structure are of crucial importance for the optimal (forward-expected) consumption pattern. At this point it can be noted that in general only the form of the term structure of interest rates matters for the optimal (forward-expected) consumption patterns for the benchmark cases of logarithmic utility investors and infinitely risk averse investors, as described in Corollary 1 and Corollary 2.

\section{Specific examples}

In this section we describe two specific examples and present numerical results on how to hedge multi-factor interest rate risk in a dynamic setting. The first example is based on the term structure dynamics from the Vasicek (1977) model while the second example considers a nonMarkovian three-factor HJM-term structure model where the term structure can exhibit three different kinds of changes: a parallel level change, a slope change, or a curvature change.

\subsection{Vasicek example}

In the following example we allow for utility from intermediate consumption by setting the preference parameter $K$ equal to $\frac{1}{2}$ in the specification of the utility function in (1) so that utility from intermediate consumption and utility from terminal wealth are equally weighted. On the other hand, the set-up for investment assets in the following example is basically as in Brennan and Xia (1998) and Sørensen (1999) who only consider utility from terminal wealth. The agent can thus invest in a single stock and a single bond as well as the "instantaneously" riskfree bank account. The term structure dynamics are described by the one-factor term structure model originally suggested by Vasicek (1977). In particular, the dynamics of the short-term risk-free interest rate is described by an Ornstein-Uhlenbeck process of the form,

$$
d r_{t}=\kappa\left(\theta-r_{t}\right) d t-\sigma_{r} d w_{B t}
$$

where the parameter $\theta$ describes the long-run level for the short-term interest rate, $\kappa$ is a mean-reversion parameter that determines the strength of tendency to the long-run level, and the parameter $\sigma_{r}$ describes the interest rate volatility. Besides the parameters describing the 
interest rate dynamics, the parameter denoted $\lambda_{B}$ in the context of section 3 determines the price of interest rate risk.

Using standard no-arbitrage arguments, one can solve for prices on interest rate contingent claims in the Vasicek-model. The possible forms of the term structure of forward interest rates can thus be determined by solving for prices on zero-coupon bonds. The $\tau$-maturity forward rate at time $t$ in the Vasicek-model is given by

$$
f_{t}(\tau)=e^{-\kappa(\tau-t)} r_{t}+r_{\infty}\left(1-e^{-\kappa(\tau-t)}\right)+\frac{\sigma_{r}^{2}}{2 \kappa} e^{-\kappa(\tau-t)} b(\tau-t)
$$

where

$$
\begin{aligned}
& r_{\infty}=\theta+\frac{\lambda_{B} \sigma_{r}}{\kappa}-\frac{\sigma_{r}^{2}}{2 \kappa^{2}} \\
& b(s)=\frac{1}{\kappa}\left(1-e^{-\kappa s}\right) .
\end{aligned}
$$

The dynamics of the $\tau$-maturity forward rate can be determined from (38) and (37) and an application of Ito's lemma. In particular, it is seen that the forward rate volatility structure in this example has the form $\sigma_{f}(t, \tau)=-\sigma_{r} e^{-\kappa(\tau-t)}$. Within the HJM-framework of section 3, this volatility structure and an initial term structure of forward rates of the form in (38) provide a complete specification of the Vasicek (1977) term structure model.

The agent can invest in a single stock as well as bonds and the bank account. In the specific case of a one-factor interest rate model it is sufficient that the agent can invest in a single bond besides the stock and the bank account in order to implement the complete markets optimal solution. The price process of the single stock is described in equation (25) where in this case $\sigma_{S 1}$ and $\sigma_{S 2}$ are scalars (i.e., of dimension $1 \times 1$ ).

The specific parameter values used in the following numerical example are chosen as follows:

$$
\begin{aligned}
& \theta=0.04, \kappa=0.15, \sigma_{r}=0.015, \sigma_{S 1}=0.0625, \sigma_{S 2}=0.2421 \\
& \varphi_{S}=0.05, \lambda_{S}=0.19365, \lambda_{B}=0.05
\end{aligned}
$$

In particular, the parameters $\kappa, \theta$, and $\sigma_{r}$ that describe the interest rate dynamics are chosen so that they are close to those obtained by Chan, Karolyi, Longstaff, and Sanders (1992) for the Vasicek interest rate process. The parameters for the stock process are chosen such that the expected excess return on the stock is $\varphi_{S}=5 \%$, the volatility of the stock is constant $25 \%$ $\left(=\left(\sigma_{S 1}^{2}+\sigma_{S 2}^{2}\right)^{1 / 2}\right)$, and the "instantaneous" correlation coefficient between the stock and the short-term interest rate is constant $-25 \%$ (and, hence, the correlation between the stock and any bond in the one-factor Vasicek-model is $25 \%$ ). The $5 \%$ expected rate of excess return on the stock is below the $8.4 \%$ point estimate suggested by the Ibbotson Associates 1926-1994 historical returns data on stocks (see, e.g., Brealey and Myers (1996), chapter 7, Table 7-1). Though, as 
pointed out by Brown, Goetzmann and Ross (1995), the use of realized mean returns in this context is likely to involve a survival bias which could be as high as 400 basis points per year.

The $25 \%$ volatility of the stock is slightly higher than $20.2 \%$ historical volatility estimate on the S\%P 500 index based on the Ibbotson and Associates returns data (see, e.g., Brealey and Myers (1996), chapter 7) but well in accordance with, say, volatilities on individual stocks and less diversified portfolios of stocks. Furthermore, the $25 \%$ positive correlation between the stock and bonds is consistent with the empirical results in, e.g., Campbell (1987), Fama and French (1989), and Shiller and Beltratti (1992). Finally, the risk premia on bonds, $\lambda_{B}=0.05$, is set so that, e.g., the expected excess return on a 10-year zero-coupon bond in the Vasicek-model is $0.39 \%{ }^{4}$

The above parameter values imply that an agent with logarithmic utility invests an $80 \%$ fraction of wealth in the stock, a fraction of $0 \%$ in bonds, and the residual $20 \%$ of wealth in the bank account. On the other hand, agents with non-logarithmic utility want to invest in bonds that have payoffs that match their (forward-expected) consumption pattern in order to hedge changes in the opportunity set, as described in Proposition 2. The infinitely risk averse investors invest $100 \%$ in the hedge bond while, e.g., an investor with constant relative risk aversion, $\gamma$, equal to 2 will invest $50 \%$ of wealth in the speculative portfolio and $50 \%$ of wealth in the hedge bond; i.e. the portfolio composition in this case is: $40 \%$ in the stock, $10 \%$ in the bank account, and $50 \%$ in the hedge bond.

We will consider three cases with different initial term structures of forward rates. These three forms are given by setting the short-term interest rate equal to 0.01, 0.04, and 0.07, respectively. The three forms of the initial term structure of forward interest rates are displayed in Figure 1.

\section{[ INSERT FIGURE 1 ABOUT HERE ]}

As formalized in Proposition 2, the (forward-expected) consumption pattern of the agent is crucial for how to hedge changes in interest rates. The (forward-expected) consumption pattern and the (forward-expected) terminal wealth of the agent can be determined by inserting in the expressions in (35) and (36). In particular, the consumption pattern over time depends on the term structure of forward rates through the occurrence of the zero-coupon price $P(t, \tau)$ $=\exp \left(-\int_{t}^{\tau} f(t, s) d s\right)$ in the expressions. Also, the consumption pattern over time depend on the prices on risk in the economy through the expression for the variance of the log-pricing kernel, $g(t, s)$ in (33). Using that the zero-coupon bond volatility is $\sigma_{P}(t, \tau)=-\int_{t}^{\tau} \sigma_{f}(t, u) d u$

\footnotetext{
${ }^{4}$ Again, Brealey and Myers (1996, chapter 7) tabulate the average historical excess return on government bonds to be slightly higher, $1.4 \%$, based on the Ibbotson and Associates returns data.
} 
$=\sigma_{r} b(\tau-t)$ and by evaluating the integrals in (33), one obtains

$$
g(t, s)=\left(\lambda_{B}^{2}+\lambda_{S}^{2}\right)(s-t)+2\left(r_{\infty}-\theta\right)(b(s-t)-(s-t))-\frac{\sigma_{r}^{2}}{2 \kappa}(b(s-t))^{2}
$$

The (forward-expected) consumption patterns are displayed in Figure 2 to Figure 6 for different degrees of relative risk aversion and for a subjective time discount rate of $\beta=0.03$ and time horizon of $T=25$ (years).

\section{[ INSERT FIGURE 2 TO FIGURE 6 ABOUT HERE ]}

The consumption patterns in the figures describe the specific payment schedules for the relevant coupon-bonds that the different investors should use in order to hedge changes in the term structure of interest rates. The log-utility investors and the infinitely risk averse investors are polar benchmark cases where either the demand for the hedge bond is exactly $0 \%$ or exactly $100 \%$. Investors in between these two polar cases will invest a fraction of wealth between $0 \%$ and $100 \%$ in the specific bonds in order to hedge changes in the opportunity set. For example, investors with relative risk aversion of $4 / 3,2$, and 4 should optimally invest $25 \%, 50 \%$, and $75 \%$, respectively, in their specific hedge bonds.

For a log-utility investor and for an investor with $\gamma=\infty$, the forward-expected consumption and terminal wealth patterns, as stated in the general expressions in Corollary 1 and Corollary 2, only depend on the initial term structure of interest rates. In particular, for $\gamma=\infty$ the forwardexpected consumption pattern is always flat, as displayed in Figure 6, while the forward-expected consumption pattern for a log-utility investor in Figure 2 depends on the subjective discount rate $\beta$ and the specific form of the current term structure. From (17) it follows that in the logarithmic utility case, $\gamma=1$, the forward-expected consumption rate $k(s)$ must satisfy

$$
k^{\prime}(s)=\left(f_{t}(s)-\beta\right) k(s)
$$

and, hence, that the forward-expected consumption rate as a function of the time to consumption is increasing whenever the forward rate is higher than the subjective discount rate $\beta=0.03$, and vice versa. The consumption patterns for the investors in Figure 3 to Figure 5 are basically in between the two polar benchmark cases of investors with logarithmic utility and infinitely risk averse investors.

\subsection{A non-Markovian three-factor HJM-model}

This example features non-Markovian dynamics of the opportunity set. We consider three different initial term structures of forward rates; these are the initial term structures from the above Vasicek example, as displayed in Figure 1. 
The term structure can exhibit basically three kinds of changes: a parallel level change, a slope change, and a curvature change. Specifically, the forward rate volatility structure is assumed to have the form

$$
\sigma_{f}(t, \tau)^{\prime}=-\left(\sigma_{1}, \sigma_{2} e^{-\kappa_{2}(\tau-t)}, \sigma_{3}(\tau-t) e^{-\kappa_{3}(\tau-t)}\right), 0 \leq t \leq \tau \leq T .
$$

The dynamics of the forward rate curve is described by inserting the volatility structure (41) in (26). In particular, a change in the Wiener-process that governs movements in the first factor will result in an equal change in all forward rates for different maturities; hence, this causes a parallel level change of the forward curve. Likewise, a change in the Wiener-process that governs movements in the second factor will significantly affect forward rates with short maturities but not forward rates with long maturities; hence, this causes a slope change of the forward curve. Finally, a change in the Wiener-process that governs movements in the third factor will affect forward rates with medium maturities but neither forward rates with short and long maturities; hence, this causes a change in the curvature of the forward curve. The three factors are similar to the fundamental three components in the Nelson and Siegel (1986) structural forms widely used in practice for calibration of term structures of interest rates and also consistent with the term structure factors determined empirically by, e.g., Litterman and Scheinkman (1991).

The volatility of any zero-coupon bond is described by $\sigma_{P}(t, \tau)=-\int_{t}^{\tau} \sigma_{f}(t, u) d u$ and under the above specification of forward curve volatility, we have

$$
\sigma_{P}(t, \tau)^{\prime}=\left(\sigma_{1}(\tau-t), \sigma_{2} b_{2}(\tau-t), \frac{\sigma_{3}}{\kappa_{3}}\left(b_{3}(\tau-t)-(\tau-t) e^{-\kappa_{3}(\tau-t)}\right)\right)
$$

where $b_{j}(\tau)=\frac{1}{\kappa_{j}}\left(1-e^{-\kappa_{j} \tau}\right)$ for $j=2,3$.

As in the Vasicek-example above, it is possible to determine the optimal (forward-expected) consumption pattern and, hence, the relevant coupon bond to hedge changes in the opportunity set using the general results in Proposition 2. Besides the form of the initial term structure of interest rates the variance of the (log-) pricing kernel, $g(t, s)$, is determining the relevant consumption patterns in (35) and (36). Straightforward calculations using (33) show that

$$
\begin{aligned}
g(t, s)=\quad & \left.\lambda_{B 1}^{2}+\lambda_{B 2}^{2}+\lambda_{B 3}^{2}+\lambda_{S}^{2}\right)(s-t)-\lambda_{B 1} \sigma_{1}(s-t)^{2}+\frac{1}{3} \sigma_{1}^{2}(s-t)^{3} \\
& +\left(\frac{2 \lambda_{B 2} \sigma_{2}}{\kappa_{2}}-\frac{\sigma_{2}^{2}}{\kappa_{2}^{2}}\right)\left(b_{2}(s-t)-(s-t)\right)-\frac{\sigma_{2}^{2}}{2 \kappa_{2}}\left(b_{2}(s-t)\right)^{2} \\
& -\left(\frac{4 \lambda_{B 3} \sigma_{3}}{\kappa_{3}^{2}}-\frac{3}{2} \frac{\sigma_{3}^{2}}{\kappa_{3}^{4}}\right)(s-t)-\frac{1}{2} \frac{\sigma_{3}^{2}}{\kappa_{3}^{3}}(s-t)^{2} \\
& +\left(\frac{4 \lambda_{B 3} \sigma_{3}}{\kappa_{3}^{2}}-\frac{3}{2} \frac{\sigma_{3}^{2}}{\kappa_{3}^{4}}+\left(\frac{2 \lambda_{B 3} \sigma_{3}}{\kappa_{3}}+\frac{\sigma_{3}^{2}}{\kappa_{3}^{3}}\right)(s-t)+\frac{\sigma_{3}^{2}}{\kappa_{3}^{2}}(s-t)^{2}\right) b_{3}(s-t) \\
& -\frac{\sigma_{3}^{2}}{\kappa_{3}^{2}}\left(\frac{5}{4 \kappa_{3}}+\frac{3}{2}(s-t)+\frac{1}{2} \kappa_{3}(s-t)^{2}\right)\left(b_{3}(s-t)\right)^{2}
\end{aligned}
$$

In the following, we will tabulate numerical results for three different sets of parameters for the three-factor HJM-model. Our base case set of parameters are chosen such that the volatilities 
of short term and long term bonds as well as the expected excess returns on stocks and bonds are of the same magnitude as in the Vasicek-example above. Below, we will comment further on how this is achieved but, specifically, the parameters values in the base case are:

$$
\begin{aligned}
& \kappa_{2}=1.00, \kappa_{3}=0.50, \sigma_{1}=0.00325, \sigma_{2}=0.01184, \sigma_{3}=0.00869 \\
& \sigma_{S 1}=(0.03187,0.02305,0.04857)^{\prime}, \sigma_{S 2}=0.24206 \\
& \varphi_{S}=0.05, \lambda_{S}=0.19365, \lambda_{B}=(0.02549,0.01844,0.03886)^{\prime}
\end{aligned}
$$

In choosing the parameters in (44) we first fixed $\kappa_{2}$ and $\kappa_{3}$ so that it makes sense to talk about a slope effect and a curvature effect in the dynamics of the forward rate curve in (26). In the present context, the innovations in the forward curve are generated by a three-dimensional Wiener process, $w_{B}=\left(w_{B 1}, w_{B 2}, w_{B 3}\right)^{\prime}$. As described above, an innovation in $w_{B 1}$ affects all forward rates equally while, e.g., an innovation in $w_{B 2}$ affects short rates but not very long rates. For example, $\kappa_{2}=1.00$ implies that if an innovation in $w_{B 2}$ increases the spot rate with 100 basis point, the 1-year forward rate is increased only by $\left(100 \times e^{-\kappa_{2} \times 1}=\right) 36.79$ basis points, and the 5-year forward rate is only increased by 0.67 basis points; hence, an innovation in $w_{B 2}$ will significantly change the slope of the forward rate curve. Likewise, an innovation in $w_{B 3}$ will not affect the very near forward rates nor the very distant forward rates but will change the curvature of the forward rate curve. The maximum amplitude in the forward rate curve caused by an innovation in $w_{B 3}$ occurs for a medium distant forward rate; specifically, for $\kappa_{3}=0.50$ the maximum amplitude occurs for the $\left(1 / \kappa_{3}=\right)$ 2-year forward rate.

While the parameters $\kappa_{2}$ and $\kappa_{3}$ are specified exogenously, the forward rate volatility parameters $\sigma_{1}, \sigma_{2}$, and $\sigma_{3}$ are calibrated in order to ensure that the volatilities of zero-coupon bonds with times to maturity equal to 0.25 years, 2 years, and 10 years, respectively, are identical to those in the Vasicek example. ${ }^{5}$ Next, $\sigma_{S 1}$ and $\sigma_{S 2}$ are chosen such that the volatility on the stock is $25 \%$ and such that the correlation coefficients between the stock and any of the three term structure factors are $-25 \%$ which corresponds to the $-25 \%$ correlation between the stock and the short-term risk-free interest rate in the Vasicek example. Finally, risk premia are also calibrated to be similar to those in the Vasicek example. In particular, the expected excess return on the stock is $5 \%$ while the risk premia on bonds, as reflected in $\lambda_{B}$, are calibrated so that there are no speculative demand for bonds (also, $\left\|\lambda_{B}\right\|=0.05$, as in the Vasicek example). The portfolio choice of a logarithmic investor is, hence, to invest $80 \%$ of wealth in the stock, $0 \%$ in bonds, and $20 \%$ in the bank account, as in the Vasicek example. Likewise, other investors allocate the same fraction of wealth into the stock, the bank account, and a hedge bond as in

\footnotetext{
${ }^{5}$ This is done by equating the relevant zero-coupon bond volatilities from (42)) to those in the Vasicek example.
} 
the Vasicek example.

The speculative demand for securities in this example is exactly similar to the speculative demand in the above Vasicek example. The way the investors want to hedge changes in the opportunity set, however, may be quite different due to the more complex dynamics of the term structure of interest rates in this HJM three-factor setting. In our view, a comparison between the hedge choice in the Vasicek example and in this HJM three-factor setting using the base case parameters in (44) is relevant for addressing questions such as: (i) is the present form of the term structure of interest rates important for how to hedge changes in the opportunity set, and (ii) is the flexibility and dynamics of the term structure of interest rates important for how to hedge changes in the opportunity set?

As formalized in Proposition 2 the forward-expected consumption pattern is crucial for the hedging behavior since the appropriate bond (or bond portfolio) for hedging changes in the opportunity set is one that has a payment schedule similar to the optimal forward-expected consumption pattern. Hence, the questions above can be answered by comparing the optimal consumption patterns across the two different examples. The optimal consumption patterns are tabulated in Table 1 for investors with different degrees of relative risk aversion. As in the Vasicek example, the investors have investment horizon of 25 years, a subjective time discount rate of $\beta=0.03$, and they equally weight utility from intermediate consumption and final wealth, i.e., $K=\frac{1}{2}$ in the general utility function specification in (1).

\section{[ INSERT TABLE 1 ABOUT HERE ]}

The (forward-expected) consumption patterns for the Vasicek dynamics are exactly identical to those displayed in Figure 2 to Figure 6 in the Vasicek example above. The (forward-expected) consumption patterns for the HJM three-factor model are for the benchmark parameters in (44). Of course, the results for log-utility investors and infinitely risk averse investors in Table 1 are exactly identical since the (forward-expected) consumption patterns of these investors depend only on the current form of the term structure of interest rates, as shown in Corollary 1 and Corollary 2. However, also for investors with relative risk aversion in between these benchmark investors the differences between the consumption patterns in the Vasicek example and in the base case HJM three-factor model seems basically ignorable. The conclusion from observing similar (forward-expected) consumption patterns from the Vasicek example and the base case HJM three-factor model is that investors need not care about the dynamics of the term structure of interest rates since in both cases the investors should hedge changes in the investment opportunity set by basically buying the same coupon bond. On the other hand, the current form of the term structure is important for the optimal consumption patterns of the investors and, 
hence, important for the precise payment schedule of the relevant bond for hedging changes in the opportunity set.

In Table 2 we have tabulated results for two other sets of parameter values for the HJM three-factor model.

\section{[ INSERT TABLE 2 ABOUT HERE ]}

In the discussion of Proposition 1, it was noted that optimal consumption choices are only altered if one change parameters that enter the dynamics (or particular moments) of the pricing kernel process. Hence, e.g., changing the volatilities $\sigma_{S 1}$ and $\sigma_{S 2}$ of the investment assets will have no consequences for the optimal forward-expected consumption pattern and, hence, no consequences for the relevant coupon bond to hedge changes in the opportunity set. On the other hand, if one changes risk premia or parameters in the description of the term structure dynamics the optimal consumption pattern will in general be affected. Therefore, we only consider two other sets of parameters: one in which forward-rate volatility parameters are changed and one in which risk premia parameters are changed.

The two sets of alternative parameters considered in Table 2 are:

$$
\begin{aligned}
& \kappa_{2}=1.00, \kappa_{3}=0.50, \sigma_{1}=0.00650, \sigma_{2}=0.02367, \sigma_{3}=0.01738 \\
& \sigma_{S 1}=(0.03187,0.02305,0.04857)^{\prime}, \sigma_{S 2}=0.24206 \\
& \varphi_{S}=0.05, \lambda_{S}=0.19365, \lambda_{B}=(0.02549,0.01844,0.03886)^{\prime}
\end{aligned}
$$

and

$$
\begin{aligned}
& \kappa_{2}=1.00, \kappa_{3}=0.50, \sigma_{1}=0.00325, \sigma_{2}=0.01184, \sigma_{3}=0.00869 \\
& \sigma_{S 1}=(0.03187,0.02305,0.04857)^{\prime}, \sigma_{S 2}=0.24206 \\
& \varphi_{S}=0, \lambda_{S}=0, \lambda_{B}=(0,0,0)^{\prime}
\end{aligned}
$$

respectively.

The parameter set in (45) differs from the base set of parameters in (44) alone by higher volatilities on the forward rate curve; specifically, the parameters in (45) are chosen such that the volatilities on zero-coupon bonds with time to maturity equal to 0.25 years, 2 years, and 10 years, respectively, are exactly twice as large as in the HJM base case parameters set and, hence, twice as large as in the Vasicek example. The speculative demands for stocks and bonds are similar to those in the base case, i.e. a logarithmic utility investor invest an $80 \%$ fraction of wealth in the stock, $0 \%$ in bonds, and $20 \%$ in the bank account.

The parameter set in (46) differs from the base set of parameters in (44) alone by having zero prices on risk so that the speculative demands for stocks and bonds are zero, i.e. a logarithmic 
utility investor in this case invests a $0 \%$ fraction of wealth in the stock, $0 \%$ in bonds, and $100 \%$ in the bank account.

The optimal (forward-expected) consumption patterns in the HJM three-factor example with the above parameter choices are tabulated in Table 2 under the labels "HJM-2" and "HJM-3", respectively. The optimal (forward-expected) consumption patterns for the benchmark parameter set in (44) are identical to those in Table 1 and tabulated under the label "HJM-1" in Table 2. For the polar cases of log-utility investors and infinitely risk averse investors the optimal consumption patterns are unaltered across the different parameter sets since these only depend on the initial form of the term structure; these cases are, therefore, not tabulated in Table 2.

For investors with preferences in between the polar cases of logarithmic utility and infinite risk aversion, the (forward-expected) consumption patterns do depend on the specific set of parameters applied, as can be seen from Table 2. However, it seems that the optimal consumption patterns do not change dramatically across the different parameter sets. In particular, the consumption patterns in the case of higher forward rate volatilities are basically similar to those in the benchmark parameter case (44) and in the Vasicek-example.

In order to have an objective measure of the distance between the different consumption plans in Table 2 and, hence, of the relevant bonds to hedge changes in the opportunity set, we have also tabulated Fisher-Weil durations in Table 2. The Fisher-Weil duration measure is in this context defined by

$$
\frac{\int_{t}^{T}(s-t) k(s) P_{t}(s) d s+(T-t) k(T) P_{t}(T)}{\int_{t}^{T} k(s) P_{t}(s) d s+k(T) P_{t}(T)}
$$

and is a measure of the average time to the payments of any particular bond. Even for the case of zero risk premia, the durations of the relevant coupon bond for hedging changes in the opportunity set seem close to the relevant durations implied by the other parameter sets considered in Table 2.

\section{Conclusion}

In this paper we have derived optimal strategies for investments in stocks and term-structure derivatives for a CRRA investor in a complete market. We provided explicit result on how to hedge changes in the investment opportunity set in the case of multi-factor Gaussian HJM interest rates and deterministic market prices of risk. In particular, we have demonstrated how changes in the investment opportunity set can be hedged by a single bond: A zero-coupon bond for the case of utility from terminal wealth only and a continuous-coupon bond in the case 
of utility from intermediate consumption. Due to the limitations of the assumed dynamics of the financial markets, it is of course highly relevant to expand our study to models with more realistic term structure dynamics and other specifications of market prices of risk. We leave such an analysis to future research. 


\section{Appendix}

\section{Proof: (Proposition 1)}

At any point in time $t, 0 \leq t \leq T$, the optimal consumption and terminal wealth plan will maximize expected remaining life-time utility and, hence, solve the problem:

$$
\sup _{\left\{C_{s}, W_{T}\right\}} K \cdot \mathrm{E}_{t}\left[\int_{t}^{T} U_{1}\left(C_{s}, s\right) d s\right]+(1-K) \cdot \mathrm{E}_{t}\left[U_{2}\left(W_{T}\right)\right]
$$

subject to

$$
\mathrm{E}_{t}\left[\int_{t}^{T}\left(\frac{\zeta_{s}}{\zeta_{t}}\right) C_{s} d s+\left(\frac{\zeta_{T}}{\zeta_{t}}\right) W_{T}\right] \leq W_{t}
$$

where $U_{1}$ and $U_{2}$ are defined in (1). The first-order conditions, which the optimal consumption and terminal wealth plan must satisfy, are given by:

$$
K e^{-\beta(s-t)} C_{s}^{-\gamma}=\Psi \frac{\zeta_{s}}{\zeta_{t}}, 0 \leq t \leq s \leq T
$$

and

$$
(1-K) e^{-\beta(T-t)} W_{T}^{-\gamma}=\Psi \frac{\zeta_{T}}{\zeta_{t}}
$$

or, equivalently,

$$
C_{s}=\Psi^{-\frac{1}{\gamma}} K^{\frac{1}{\gamma}} e^{-\frac{\beta}{\gamma}(s-t)}\left(\frac{\zeta_{s}}{\zeta_{t}}\right)^{-\frac{1}{\gamma}}, 0 \leq t \leq s \leq T
$$

and

$$
W_{T}=\Psi^{-\frac{1}{\gamma}}(1-K)^{\frac{1}{\gamma}} e^{-\frac{\beta}{\gamma}(T-t)}\left(\frac{\zeta_{T}}{\zeta_{t}}\right)^{-\frac{1}{\gamma}}
$$

Inserting (49) and (50) into the budget constraint (48), we obtain

$$
W_{t}=\Psi^{-\frac{1}{\gamma}} \mathrm{E}_{t}\left[\int_{t}^{T}\left(\frac{\zeta_{s}}{\zeta_{t}}\right) K^{\frac{1}{\gamma}} e^{-\frac{\beta}{\gamma}(s-t)}\left(\frac{\zeta_{s}}{\zeta_{t}}\right)^{-\frac{1}{\gamma}} d s+\left(\frac{\zeta_{T}}{\zeta_{t}}\right)(1-K)^{\frac{1}{\gamma}} e^{-\frac{\beta}{\gamma}(T-t)}\left(\frac{\zeta_{T}}{\zeta_{t}}\right)^{-\frac{1}{\gamma}}\right]
$$

After using Fubini's Theorem to interchange the order of expectations and integration in the last term on the right hand side of this equality the term is seen to be equal to $Q_{t}$, as defined in equation (9). Hence, solving for the Lagrange multiplier $\Psi$, we have

$$
\Psi^{-\frac{1}{\gamma}}=\frac{W_{t}}{Q_{t}}
$$

Substituting the expression for $\Psi$ in (51) into (49) and (50), the optimal consumption and terminal wealth plan can be written on the form

$$
C_{s}=\frac{W_{t}}{Q_{t}} K^{\frac{1}{\gamma}} e^{-\frac{\beta}{\gamma}(s-t)}\left(\frac{\zeta_{s}}{\zeta_{t}}\right)^{-\frac{1}{\gamma}}, 0 \leq t \leq s \leq T
$$

and

$$
W_{T}=\frac{W_{t}}{Q_{t}}(1-K)^{\frac{1}{\gamma}} e^{-\frac{\beta}{\gamma}(T-t)}\left(\frac{\zeta_{T}}{\zeta_{t}}\right)^{-\frac{1}{\gamma}}
$$


The value function can now be determined by inserting the optimal consumption and terminal wealth plan in (52) and (53) into the objective function in (47). We obtain

$$
\begin{aligned}
J_{t}= & K \mathrm{E}_{t}\left[\int_{t}^{T} e^{-\beta(s-t)} \frac{C_{s}^{1-\gamma}-1}{1-\gamma} d s\right]+(1-K) \mathrm{E}_{t}\left[e^{-\beta(T-t)} \frac{W_{T}^{1-\gamma}-1}{1-\gamma}\right] \\
= & \frac{1}{1-\gamma}\left(K \int_{t}^{T} e^{-\beta(s-t)} \mathrm{E}_{t}\left[C_{s}^{1-\gamma}\right] d s+(1-K) e^{-\beta(T-t)} \mathrm{E}_{t}\left[W_{T}^{1-\gamma}\right]\right) \\
& \quad-\frac{1}{1-\gamma}\left(K \int_{t}^{T} e^{-\beta(s-t)} d s+(1-K) e^{-\beta(T-t)}\right) \\
= & \frac{1}{1-\gamma}\left(\frac{W_{t}}{Q_{t}}\right)^{1-\gamma}\left(K^{\frac{1}{\gamma}} \int_{t}^{T} e^{-\frac{\beta}{\gamma}(s-t)} \mathrm{E}_{t}\left[\left(\frac{\zeta_{s}}{\zeta_{t}}\right)^{\frac{\gamma-1}{\gamma}}\right] d s+(1-K)^{\frac{1}{\gamma}} e^{-\frac{\beta}{\gamma}(T-t)} \mathrm{E}_{t}\left[\left(\frac{\zeta_{T}}{\zeta_{t}}\right)^{\frac{\gamma-1}{\gamma}}\right]\right) \\
& \quad-\frac{1}{1-\gamma} A(t) \\
= & \frac{Q_{t}^{\gamma} W_{t}^{1-\gamma}-A(t)}{1-\gamma}
\end{aligned}
$$

where we have used Fubini's Theorem and the definition of $A(t)$ in the third equality. The fourth equality follows by using the definition of $Q_{t}$ in (9). This proves the form of the value function stated in (11).

The optimal consumption choice stated in (12) follows as the special case where $s=t$ in the expression for the optimal consumption plan in (52).

Finally, it remains to be shown that the optimal portfolio policy is as stated in (13). Since the optimal plan for optimal terminal wealth in (53) is valid for any $t, 0 \leq t \leq T$, and in particular also for $t=0$, we have that

$$
\frac{W_{0}}{Q_{0}}(1-K)^{\frac{1}{\gamma}} e^{-\frac{\beta}{\gamma} T}\left(\frac{\zeta_{T}}{\zeta_{0}}\right)^{-\frac{1}{\gamma}}=\frac{W_{t}}{Q_{t}}(1-K)^{\frac{1}{\gamma}} e^{-\frac{\beta}{\gamma}(T-t)}\left(\frac{\zeta_{T}}{\zeta_{t}}\right)^{-\frac{1}{\gamma}}
$$

or, equivalently,

$$
W_{t}=W_{0} e^{-\frac{\beta}{\gamma} t}\left(\frac{Q_{t}}{Q_{0}}\right)\left(\frac{\zeta_{t}}{\zeta_{0}}\right)^{-\frac{1}{\gamma}}
$$

Using the expression for optimal wealth in (54), and by an application of Ito's lemma, (10), and (4), it follows that

$$
d W_{t}=W_{t}\left((\ldots) d t+\sigma_{Q t} d w_{t}+\left(\frac{1}{\gamma}\right) \lambda_{t} d w_{t}\right)
$$

In order to replicate the dynamics of optimally invested wealth in (55) the diffusion coefficients must be matched (almost surely). Note that the drift term has been left unspecified since, using the martingale solution approach, this term will automatically be matched once the diffusion coefficients are matched. The claimed portfolio policy in (13) can now be verified by inspection; i.e., by inserting the portfolio policy in (13) into (6) it is seen that the proposed strategy ensures a match with the diffusion coefficients in (55). This ends the proof. 
Proof: (Corollary 1)

The proofs of (14), (15), and (16) follow exactly the same steps as in the proof of Proposition 1 above and, hence, will not be repeated here.

The analog to the optimal consumption plan in (52) is given by setting $\gamma$ equal to one,

$$
C_{s}=\frac{W_{t}}{Q_{t}} K e^{-\beta(s-t)}\left(\frac{\zeta_{s}}{\zeta_{t}}\right)^{-1}=C_{t} e^{-\beta(s-t)}\left(\frac{\zeta_{s}}{\zeta_{t}}\right)^{-1}, 0 \leq t \leq s \leq T
$$

where the last equality follows from (15). The forward-expected consumption rate at time $s$ is by definition given by

$$
P_{t}(s) \hat{\mathrm{E}}_{t}^{s}\left[C_{s}\right]=\mathrm{E}_{t}\left[\left(\frac{\zeta_{s}}{\zeta_{t}}\right) C_{s}\right]=C_{t} e^{-\beta(s-t)}
$$

where we have used (56) to obtain the last equality. The form of the forward-expected consumption rates in (17) now follow by solving for $\hat{\mathrm{E}}_{t}^{s}\left[C_{s}\right]$. The proof of the expression for forwardexpected terminal wealth in (18) follows analogously (in this case by using the pendant to optimal terminal wealth in (53) for $\gamma=1)$.

\section{Proof: (Corollary 2)}

The limiting optimal consumption plan is given by the limit of (52),

$$
C_{s}=\frac{W_{t}}{Q_{t}}, 0 \leq t \leq s \leq T .
$$

Since (57) is valid for any $s$ and $t$, and especially for $t=0$, it follows that the process $C_{s}, s \geq 0$, is constant. The claim in (19) follows directly from (57) as do the claim in (22). Taking the limit of (53) as $\gamma$ approaches infinity, it is seen that $W_{T}=W_{t} / Q_{t}=C_{t}$; the proves the claim in $(23)$.

The portfolio policy in (20) is obtained as the limit of (13) as $\gamma$ approaches infinity.

The expression for $Q_{t}$ in (21) can be obtained as the limit of the expression in (9) as $\gamma$ approaches infinity. ${ }^{6}$ Alternatively, it follows from (19) that $Q_{t}$ is proportional to the current wealth, $W_{t}$. Since current wealth must be invested in an annuity (which ensures a "flat" payment schedule equal to the optimal consumption plan $C_{t}=C_{0}$ ), the present value of this annuity must always be: $W_{t}=C_{t} \cdot\left(\int_{t}^{T} P_{t}(s) d s+P_{t}(T)\right)$. The claimed form of $Q_{t}$ in (21) now follows from (19).

In the proof of Proposition 2 we make use of the following Leibnitz-type rule for stochastic processes:

\footnotetext{
${ }^{6}$ In this case it is implicitly assumed that the relevant expectations of the pricing kernel are sufficiently wellbehaved so that a convergence theorem can be applied to interchange the order of integration and passage to the limit.
} 
Lemma 1 Let $Z_{t}(s)$ be a family of stochastic processes such that for each fixed $s \in[0, T]$

$$
d Z_{t}(s)=\mu_{t}(s) d t+\sigma_{t}(s) d w_{t}, 0 \leq t \leq s
$$

where $\sigma_{t}(s)$ satisfies

$$
\begin{aligned}
& \text { (a) } \int_{0}^{T} \sigma_{t}^{2}(s) d t<\infty \text { for all } s \in[0, T], \\
& \text { (b) } \int_{0}^{T}\left[\int_{t}^{T} \sigma_{t}(s) d s\right]^{2} d t<\infty
\end{aligned}
$$

almost surely. Let $Y_{t}$ be defined by

$$
Y_{t}=\int_{t}^{T} Z_{t}(s) d s
$$

Then the dynamics of $Y_{t}$ are given by

$$
d Y_{t}=\left[\left(\int_{t}^{T} \mu_{t}(s) d s\right)-Z_{t}(t)\right] d t+\left(\int_{t}^{T} \sigma_{t}(s) d s\right) d w_{t} .
$$

Proof: The proof is an application of the generalized Fubini-type rule for stochastic processes stated and applied in the Appendix of Heath, Jarrow and Morton (1992). Let $t_{0} \leq t_{1}$, then since

$$
Z_{t_{1}}(s)=Z_{t_{0}}(s)+\int_{t_{0}}^{t_{1}} \mu_{t}(s) d t+\int_{t_{0}}^{t_{1}} \sigma_{t}(s) d w_{t}
$$

we have

$$
\begin{aligned}
Y_{t_{1}}= & \int_{t_{1}}^{T} Z_{t_{0}}(s) d s+\int_{t_{1}}^{T}\left[\int_{t_{0}}^{t_{1}} \mu_{t}(s) d t\right] d s+\int_{t_{1}}^{T}\left[\int_{t_{0}}^{t_{1}} \sigma_{t}(s) d w_{t}\right] d s \\
= & \int_{t_{1}}^{T} Z_{t_{0}}(s) d s+\int_{t_{0}}^{t_{1}}\left[\int_{t_{1}}^{T} \mu_{t}(s) d s\right] d t+\int_{t_{0}}^{t_{1}}\left[\int_{t_{1}}^{T} \sigma_{t}(s) d s\right] d w_{t} \\
= & Y_{t_{0}}+\int_{t_{0}}^{t_{1}}\left[\int_{t}^{T} \mu_{t}(s) d s\right] d t+\int_{t_{0}}^{t_{1}}\left[\int_{t}^{T} \sigma_{t}(s) d s\right] d w_{t} \\
& \quad-\int_{t_{0}}^{t_{1}} Z_{t_{0}}(s) d s-\int_{t_{0}}^{t_{1}}\left[\int_{t}^{t_{1}} \mu_{t}(s) d s\right] d t-\int_{t_{0}}^{t_{1}}\left[\int_{t}^{t_{1}} \sigma_{t}(s) d s\right] d w_{t} \\
= & Y_{t_{0}}+\int_{t_{0}}^{t_{1}}\left[\int_{t}^{T} \mu_{t}(s) d s\right] d t+\int_{t_{0}}^{t_{1}}\left[\int_{t}^{T} \sigma_{t}(s) d s\right] d w_{t} \\
& \quad-\int_{t_{0}}^{t_{1}} Z_{t_{0}}(s) d s-\int_{t_{0}}^{t_{1}}\left[\int_{t_{0}}^{s} \mu_{t}(s) d t\right] d s-\int_{t_{0}}^{t_{1}}\left[\int_{t_{0}}^{s} \sigma_{t}(s) d w_{t}\right] d s \\
= & Y_{t_{0}}+\int_{t_{0}}^{t_{1}}\left[\int_{t}^{T} \mu_{t}(s) d s\right] d t+\int_{t_{0}}^{t_{1}}\left[\int_{t}^{T} \sigma_{t}(s) d s\right] d w_{t} \\
& \quad-\int_{t_{0}}^{t_{1}}\left[Z_{t_{0}}(s)+\int_{t_{0}}^{s} \mu_{t}(s) d t+\int_{t_{0}}^{s} \sigma_{t}(s) d w_{t}\right] d s \\
& \int_{t_{0}}^{t_{1}}\left[\int_{t}^{T} \mu_{t}(s) d s\right] d t+\int_{t_{0}}^{t_{1}}\left[\int_{t}^{T} \sigma_{t}(s) d s\right] d w_{t}-\int_{t_{0}}^{t_{1}} Z_{t}(t) d t \\
= & \left.Y_{t_{0}}+{ }_{t}\right]
\end{aligned}
$$


where the Fubini rule is used in the second and fourth equality while the first equality follows by inserting (58) in the definition of $Y_{t}$ and, also, the last equality follows by using (58) and the fact that $\int_{t_{0}}^{t_{1}} Z_{t}(t) d t=\int_{t_{0}}^{t_{1}} Z_{s}(s) d s$; the other equalities follow by pure manipulation of the involved expressions. The claim now follows.

\section{Proof: (Proposition 2)}

In order to prove the form of the value function and the optimal consumption strategy, we only need to verify that under the specific assumptions $Q_{t}$, as defined in equation (9), has the form stated in (30), (31), (32), and (33).

It follows from (9) that $Q_{t}$ can always be written on the form in (30) with

$$
\begin{aligned}
& Z_{t}(s)=K^{\frac{1}{\gamma}} e^{-\frac{\beta}{\gamma}(s-t)} \mathrm{E}_{t}\left[\left(\frac{\zeta_{s}}{\zeta_{t}}\right)^{\frac{\gamma-1}{\gamma}}\right], 0 \leq t \leq s<T \\
& Z_{t}(T)=(1-K)^{\frac{1}{\gamma}} e^{-\frac{\beta}{\gamma}(T-t)} \mathrm{E}_{t}\left[\left(\frac{\zeta_{T}}{\zeta_{t}}\right)^{\frac{\gamma-1}{\gamma}}\right] .
\end{aligned}
$$

Using the assumption that $\lambda(t)$ and the forward volatilities $\sigma_{f}(t, \tau)$ are deterministic functions of time, it follows that the pricing kernel $\zeta_{t}$ in (3) is lognormally distributed and, hence, it is possible to compute in closed-form the expectations that enter the expressions for $Z_{t}(s)$ and $Z_{t}(T)$ in (59) and (60). First note that the price of a zero-coupon bond must satisfy

$$
P_{t}(s)=\mathrm{E}_{t}\left[\left(\frac{\zeta_{s}}{\zeta_{t}}\right)\right]=\exp \left\{\mathrm{E}_{t}\left[\ln \left(\frac{\zeta_{s}}{\zeta_{t}}\right)\right]+\frac{1}{2} \operatorname{Var}_{t}\left[\ln \left(\frac{\zeta_{s}}{\zeta_{t}}\right)\right]\right\}
$$

It follows that

$$
\begin{aligned}
\mathrm{E}_{t}\left[\left(\frac{\zeta_{s}}{\zeta_{t}}\right)^{\frac{\gamma-1}{\gamma}}\right] & =\exp \left\{\frac{\gamma-1}{\gamma} \mathrm{E}_{t}\left[\ln \left(\frac{\zeta_{s}}{\zeta_{t}}\right)\right]+\frac{1}{2}\left(\frac{\gamma-1}{\gamma}\right)^{2} \operatorname{Var}_{t}\left[\ln \left(\frac{\zeta_{s}}{\zeta_{t}}\right)\right]\right\} \\
& =P_{t}(s)^{\frac{\gamma-1}{\gamma}} \exp \left\{\frac{1-\gamma}{2 \gamma^{2}} g(t, s)\right\}, 0 \leq t \leq s \leq T
\end{aligned}
$$

where for notational simplicity we have introduced the deterministic function

$$
g(t, s)=\operatorname{Var}_{t}\left[\ln \left(\frac{\zeta_{s}}{\zeta_{t}}\right)\right],
$$

which can be rewritten using (3) and (27) as

$$
\begin{aligned}
g(t, s) & =\operatorname{Var}_{t}\left[-\int_{t}^{s} r_{u} d u-\frac{1}{2} \int_{t}^{s}\|\lambda(u)\|^{2} d u-\int_{t}^{s} \lambda(u)^{\prime} d w_{u}\right] \\
& =\int_{t}^{s}\|\lambda(u)\|^{2} d u+\int_{t}^{s}\left\|\int_{u}^{s} \sigma_{f}(u, \tau) d \tau\right\|^{2} d u+2 \int_{t}^{s} \lambda_{B}(u)^{\prime}\left[\int_{u}^{s} \sigma_{f}(u, \tau) d \tau\right] d u \\
& =\int_{t}^{s}\|\lambda(u)\|^{2} d u+\int_{t}^{s}\left\|\sigma_{P}(u, s)\right\|^{2} d u-2 \int_{t}^{s} \lambda_{B}(u)^{\prime} \sigma_{P}(u, s) d u
\end{aligned}
$$


The stated forms of $Z_{t}(s)$ and $Z_{t}(T)$ in (31) and (32) now follow by substituting (61) into (59) and (60).

We will now prove that the optimal portfolio policy is as stated in (34). Note that by using the general expression for the portfolio policy in Proposition 1, we need only to show that $\sigma_{Q t}=\left(\frac{\gamma-1}{\gamma}\right)\left(\sigma_{B_{t}^{\text {cpn }}}^{\prime}, 0^{\prime}\right)^{\prime}$. We will start by proving the formulas for forward-expected consumption and terminal wealth stated in (35) and (36). By definition of the forward-expected (or certainty equivalent) payoff of the stochastic consumption rate $C_{s}, 0 \leq t \leq s<T$, we have

$$
P_{t}(s) \hat{\mathrm{E}}_{t}^{s}\left[C_{s}\right]=\mathrm{E}_{t}\left[\left(\frac{\zeta_{s}}{\zeta_{t}}\right) C_{s}\right]
$$

Inserting the optimal consumption plan derived in the proof of Proposition 1 and stated in equation (52), we obtain

$$
\begin{aligned}
P_{t}(s) \hat{\mathrm{E}}_{t}^{s}\left[C_{s}\right] & =\frac{W_{t}}{Q_{t}} K^{\frac{1}{\gamma}} e^{-\frac{\beta}{\gamma}(s-t)} \mathrm{E}_{t}\left[\left(\frac{\zeta_{s}}{\zeta_{t}}\right)^{\frac{\gamma-1}{\gamma}}\right] \\
& =\frac{W_{t}}{Q_{t}} Z_{t}(s)
\end{aligned}
$$

where the second equality follows using (59). The formula for the forward-expected consumption rate in (35) now follows by inserting the expression for $Z_{t}(s)$ in (31) (which was proved above) into (63) and solving for $\hat{\mathrm{E}}_{t}^{s}\left[C_{s}\right]$. The analogous formula for $\hat{\mathrm{E}}_{t}^{T}\left[W_{T}\right]$ in (36) can be verified using exactly the same steps.

We will now show that $\sigma_{Q t}=\left(\frac{\gamma-1}{\gamma}\right)\left(\sigma_{B_{t}^{\text {cpn }}}^{\prime}, 0^{\prime}\right)^{\prime}$. First note that by an application of Ito's lemma, (31), and (28), the dynamics of $Z_{t}(s)$ can be described on the form

$$
d Z_{t}(s)=Z_{t}(s)\left((\ldots) d t+\left(\frac{\gamma-1}{\gamma}\right) \sigma_{P}(t, s)^{\prime} d w_{B t}\right)
$$

where we have left the drift term unspecified since it is of no importance for the proof. Now, since $Q_{t}$ is of the form in (30), we can apply the Leibnitz-type rule for stochastic processes stated in Lemma 1 in order to find the dynamics of $Q_{t}$. We find that

$$
d Q_{t}=(\ldots) d t+\left(\frac{\gamma-1}{\gamma}\right)\left(\int_{t}^{T} Z_{t}(s) \sigma_{P}(t, s) d s+Z_{t}(T) \sigma_{t}(T)\right)^{\prime} d w_{B t}
$$

In particular, the dynamics of $Q_{t}$ is of the general form stated in (10) with

$$
\sigma_{Q t}=\left(\frac{\gamma-1}{\gamma}\right)\left(\frac{\left(\int_{t}^{T} Z_{t}(s) \sigma_{P}(t, s) d s+Z_{t}(T) \sigma_{t}(T)\right)^{\prime}}{\int_{t}^{T} Z_{t}(s) d s+Z_{t}(T)}, 0^{\prime}\right)^{\prime} .
$$

From (63) we have that $Z_{t}(s)=\left(\frac{Q_{t}}{W_{t}}\right) P_{t}(s) k(s)$ where by definition $k(s) \equiv \hat{\mathrm{E}}_{t}^{s}\left[C_{s}\right]$. Inserting this in (65) and dividing through by the constant $\left(\frac{Q_{t}}{W_{t}}\right)$ in the numerator and denominator, the 
expression for $\sigma_{Q t}$ in (65) takes the form $\left(\frac{\gamma-1}{\gamma}\right)\left(\sigma_{B_{t}^{\text {cpn }}}^{\prime}, 0^{\prime}\right)^{\prime}$ with $\sigma_{B_{t}^{\mathrm{cpn}}}$ on the form in (29). This is the claimed portfolio policy and, hence, this ends the proof. 


\section{References}

Amin, K. I. \& Jarrow, R. A. (1992). "Pricing Options on Risky Assets in a Stochastic Interest Rate Economy". Mathematical Finance, 2, 217-237.

Brace, A. \& Musiela, M. (1994). "A Multifactor Gauss Markov Implementation of Heath, Jarrow, and Morton". Mathematical Finance, 4(3), 259-283.

Brealey, R. A. \& Myers, S. C. (1996). Principles of Corporate Finance. McGraw-Hill, 5 edition.

Brennan, M. J.; Schwartz, E. S., \& Lagnado, R. (1997). "Strategic Asset Allocation". Journal of Economic Dynamics and Control, 21, 1377-1403.

Brennan, M. J. \& Xia, Y. (1998). "Resolution of a Financial Puzzle". Working paper, John E. Anderson Graduate School of Management, UCLA.

Brown, S. J.; Goetzmann, W. N., \& Ross, S. A. (1995). "Survival". Journal of Finance, 50, $853-873$.

Campbell, J. Y. (1987). "Stock Returns and the Term Structure". Journal of Financial Economics, 18, 373-399.

Campbell, J. Y. (2000). "Asset Pricing at the Millennium". Journal of Finance, 55(4), 15151567.

Campbell, J. Y. \& Viceira, L. M. (1998). "Who Should Buy Long-Term Bonds?”. Working paper no. 6801, NBER.

Campbell, J. Y. \& Viceira, L. M. (1999). "Consumption and Portfolio Decisions when Expected Returns are Time-Varying". The Quarterly Journal of Economics, 114, 433-495.

Carverhill, A. (1994). "When is the Short Rate Markovian?". Mathematical Finance, 4(4), $305-312$.

Chacko, G. \& Viceira, L. M. (1999). "Dynamic Consumption and Portfolio Choice with Stochastic Volatility in Incomplete Markets". Working paper, Harvard University.

Chan, K. C.; Karolyi, G. A.; Longstaff, F. A., \& Sanders, A. B. (1992). "An Empirical Comparison of Alternative Models of the Short-Term Interest Rate". Journal of Finance, 47(3), 1209-1227.

Cox, J. C. \& Huang, C.-F. (1989). "Optimal Consumption and Portfolio Policies when Asset Prices follow a Diffusion Process". Journal of Economic Theory, 49, 33-83. 
Cox, J. C. \& Huang, C.-F. (1991). "A Variational Problem Arising in Financial Economics". Journal of Mathematical Economics, 20, 465-487.

Detemple, J.; Garcia, R., \& Rindisbacher, M. (1999). "A Monte-Carlo Method for Optimal Portfolios". Working paper.

Duffie, J. D. (1996). Dynamic Asset Pricing Theory. Princeton University Press, Princeton, New Jersey, 2 edition.

Duffie, J. D. \& Huang, C.-F. (1985). "Implementing Arrow-Debreu Equillibria by Continuous Trading of Few Long-Lived Securities". Econometrica, 53, 1337-1356.

Fama, E. F. \& French, K. R. (1989). "Business Conditions and Expected Returns on Stocks and Bonds". Journal of Financial Economics, 25, 23-49.

Geman, H. (1989). "The Importance of the Forward Neutral Probability in a Stochastic Approach of Interest Rates". Working paper, ESSEC.

Heath, D.; Jarrow, R., \& Morton, A. (1992). "Bond Pricing and the Term Structure of Interest Rates: A New Methodology for Contingent Claims Valuation”. Econometrica, 60(1), 77105.

Hull, J. C. (2000). Options, Futures, and other Derivatives. Prentice-Hall, 4 edition.

Ibbotson Associates (1995). "Stocks, Bonds, Bills, and Inflation: 1995 Yearbook". Chicago: Ibbotson Associates.

Ingersoll, Jr., J. E. (1987). Theory of Financial Decision Making. Studies in Financial Economics. Rowman \& Littlefield, 1 edition.

Jamshidian, F. (1987). "Pricing of Contingent Claims in the One Factor Term Structure Model". Working paper, Merrill Lynch Capital Markets.

Jamshidian, F. (1989). "An Exact Bond Option Formula". Journal of Finance, 44(1), 205-209.

Karatzas, I.; Lehoczky, J. P., \& Shreve, S. E. (1987). "Optimal Portfolio and Consumption Decisions for a "small Investor" on a Finite Horizon". SIAM Journal on Control and Optimization, 25(6), 1557-1586.

Karatzas, I. \& Shreve, S. E. (1988). Brownian Motion and Stochastic Calculus. New York: Springer-Verlag. 
Kim, T. S. \& Omberg, E. (1996). "Dynamic Nonmyopic Portfolio Behavior". Review of Financial Studies, 9(1), 141-161.

Litterman, R. \& Scheinkman, J. (1991). "Common Factors Affecting the Bond Returns". Journal of Fixed Income, 1, 54-61.

Liu, J. (1999). "Portfolio Selection in Stochastic Enviroments". Working paper, Stanford University.

Merton, R. C. (1969). "Lifetime Portfolio Selection Under Uncertainty: The Continuous-Time Case". Review of Economics and Statistics, 51, 247-257.

Merton, R. C. (1971). "Optimum Consumption and Portfolio Rules in a Continuous-Time Model". Journal of Economic Theory, 3, 373-413.

Merton, R. C. (1973). "An Intertemporal Capital Asset Pricing Model”. Econometrica, 41, $867-887$.

Merton, R. C. (1993). Continuous-time Finance. Basil Blackwell, revised edition.

Nelson, C. R. \& Siegel, A. F. (1987). "Parsimonious Modeling of Yield Curves". Journal of Business, 60, 473-489.

Ocone, D. L. \& Karatzas, I. (1991). “A Generalized Clark Representation Formula, with Application to Optimal Portfolios". Stochastics and Stochastic Report, 34, 187-220.

Shiller, R. J. \& Beltratti, A. E. (1992). "Stock prices and bond yields: Can their comovements be explained in terms of present value models?". Journal of Monetary Economics, 30, 25-46.

Sørensen, C. (1999). "Dynamic Asset Allocation and Fixed Income Management". Journal of Financial and Quantitative Analysis, 34(4), 513-531.

Vasicek, O. A. (1977). "An Equilibrium characterization of the Term Structure". Journal of Financial Economics, 5, 177-188.

Wachter, J. A. (1999). "Risk Aversion and Allocation to Long-Term Bonds". Working paper, Graduate School of Business Administration, Harvard University. 


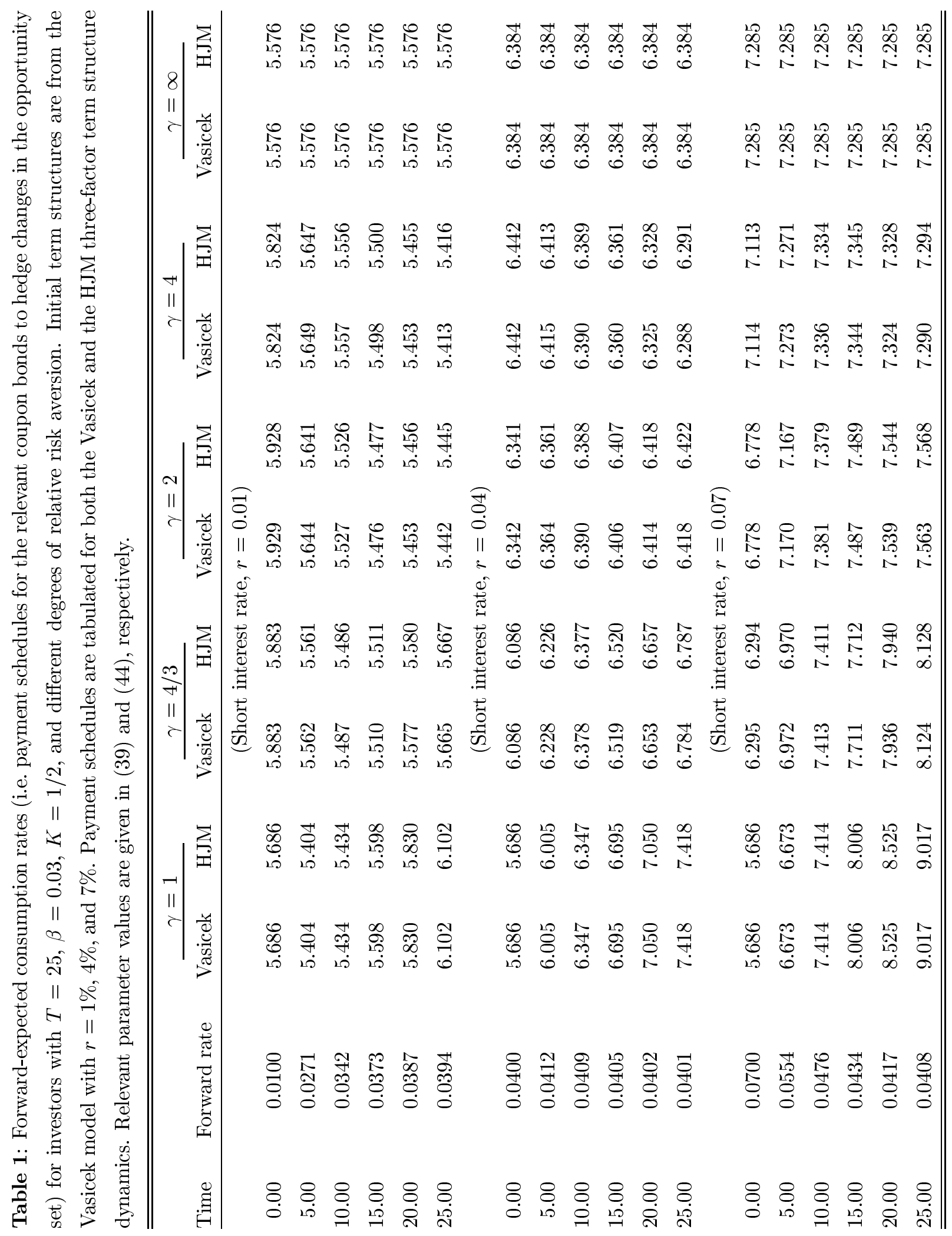




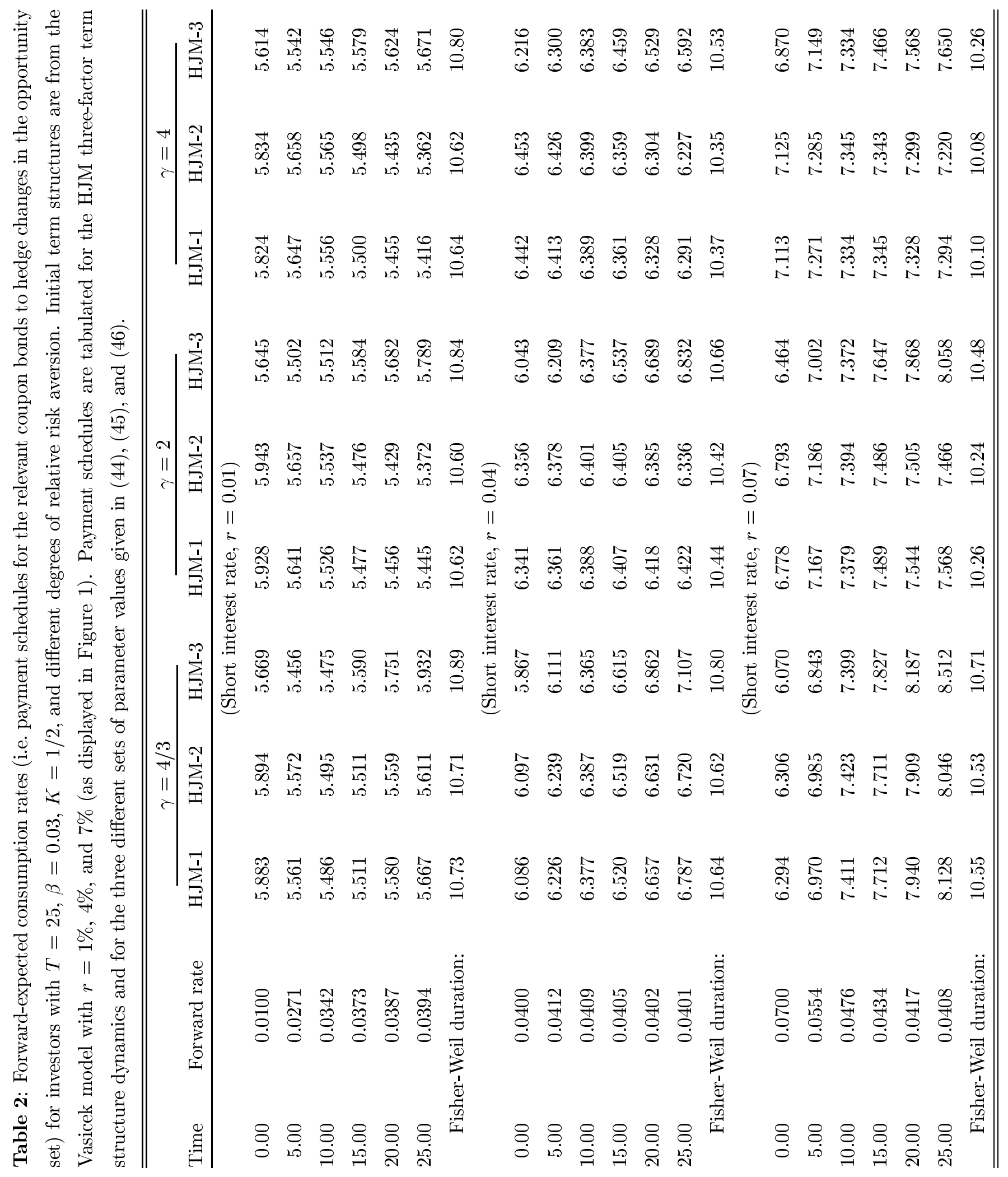




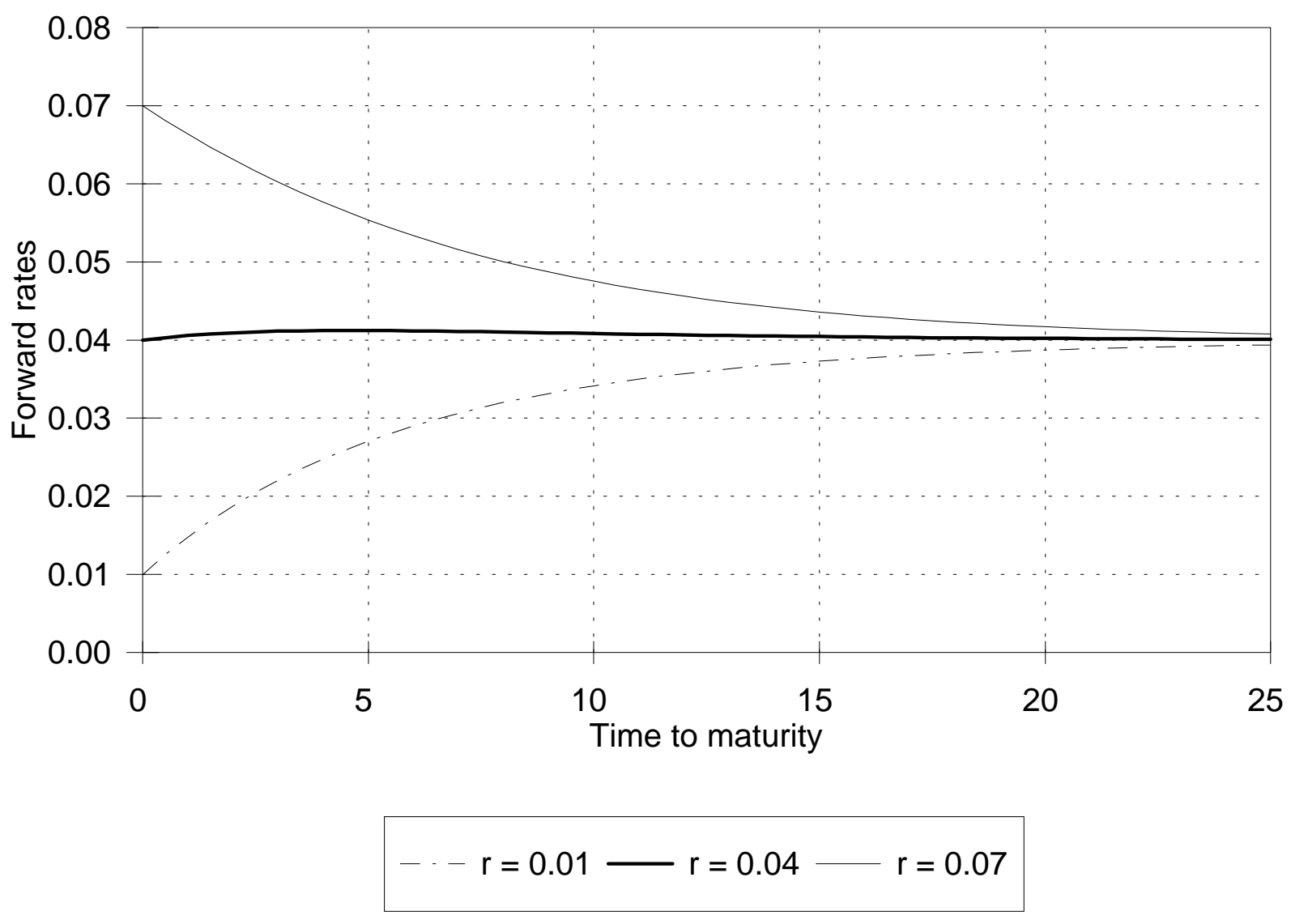

Figure 1: Term structures of forward interest rates. The figure displays forward rates as a function of time to maturity for different Vasicek term structures described by short interest rate levels of $0.01,0.04$, and 0.07 , respectively. 


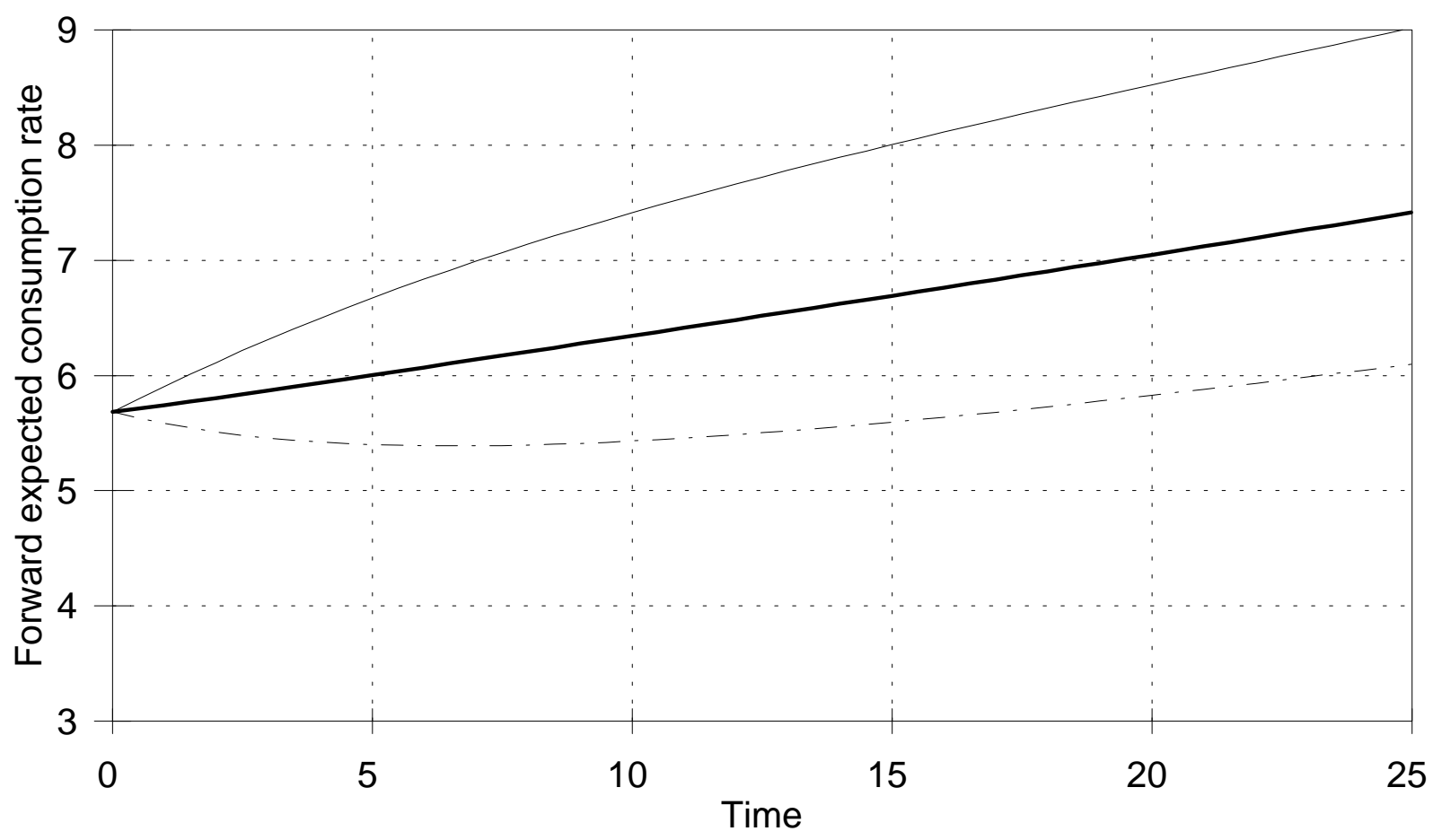

$$
-\cdot r=0.01-r=0.04-r=0.07
$$

Figure 2: Expected consumption patterns for an investor with constant relative risk aversion, $\mathbf{R R A}=\mathbf{1 . 0 0}$, and time horizon $T=\mathbf{2 5}$. The figure displays the expected consumption streams under the forward-adjusted martingale measures for the three different Vasicek term structures of forward interest rates displayed in Figure 1. 


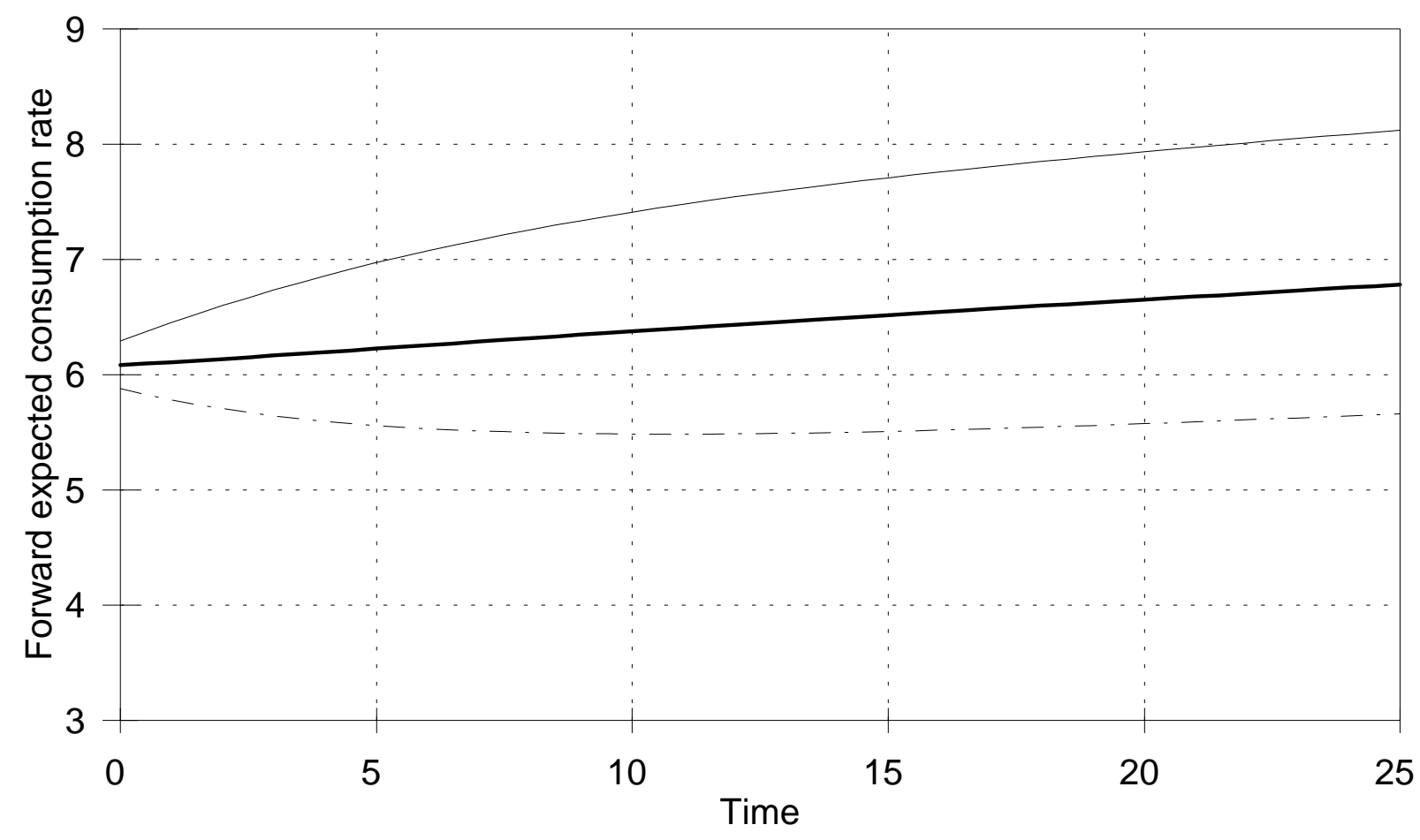

$$
--r=0.01-r=0.04-r=0.07
$$

Figure 3: Expected consumption patterns for an investor with constant relative risk aversion, $\mathbf{R R A}=\mathbf{1 . 3 3}$ and time horizon $T=\mathbf{2 5}$. The figure displays the expected consumption streams under the forward-adjusted martingale measures for the three different Vasicek term structures of forward interest rates displayed in Figure 1. 


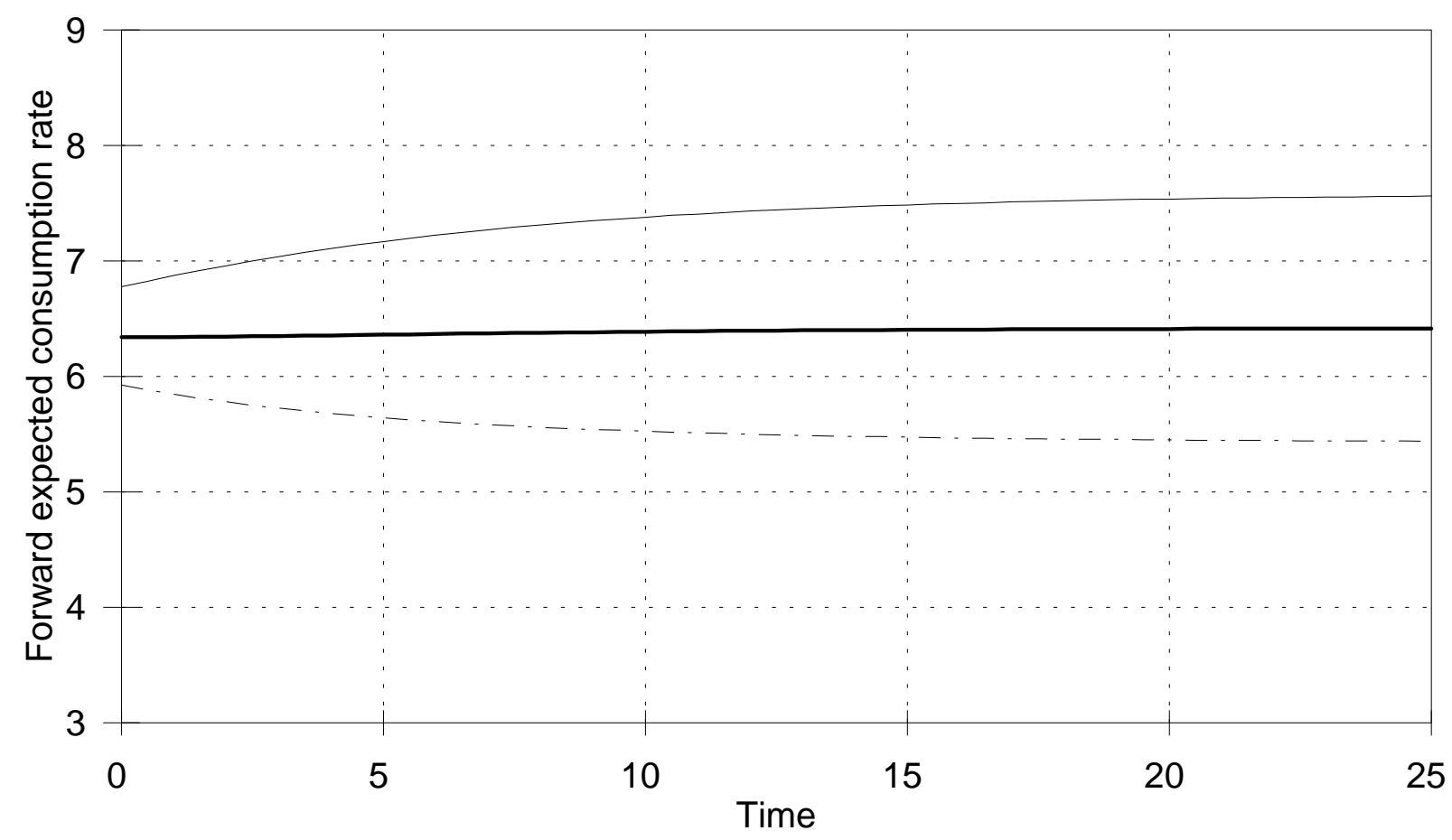

$$
-r=0.01-r=0.04-r=0.07
$$

Figure 4: Expected consumption patterns for an investor with constant relative risk aversion, $\mathbf{R R A}=\mathbf{2 . 0 0}$ and time horizon $T=\mathbf{2 5}$. The figure displays the expected consumption streams under the forward-adjusted martingale measures for the three different Vasicek term structures of forward interest rates displayed in Figure 1. 


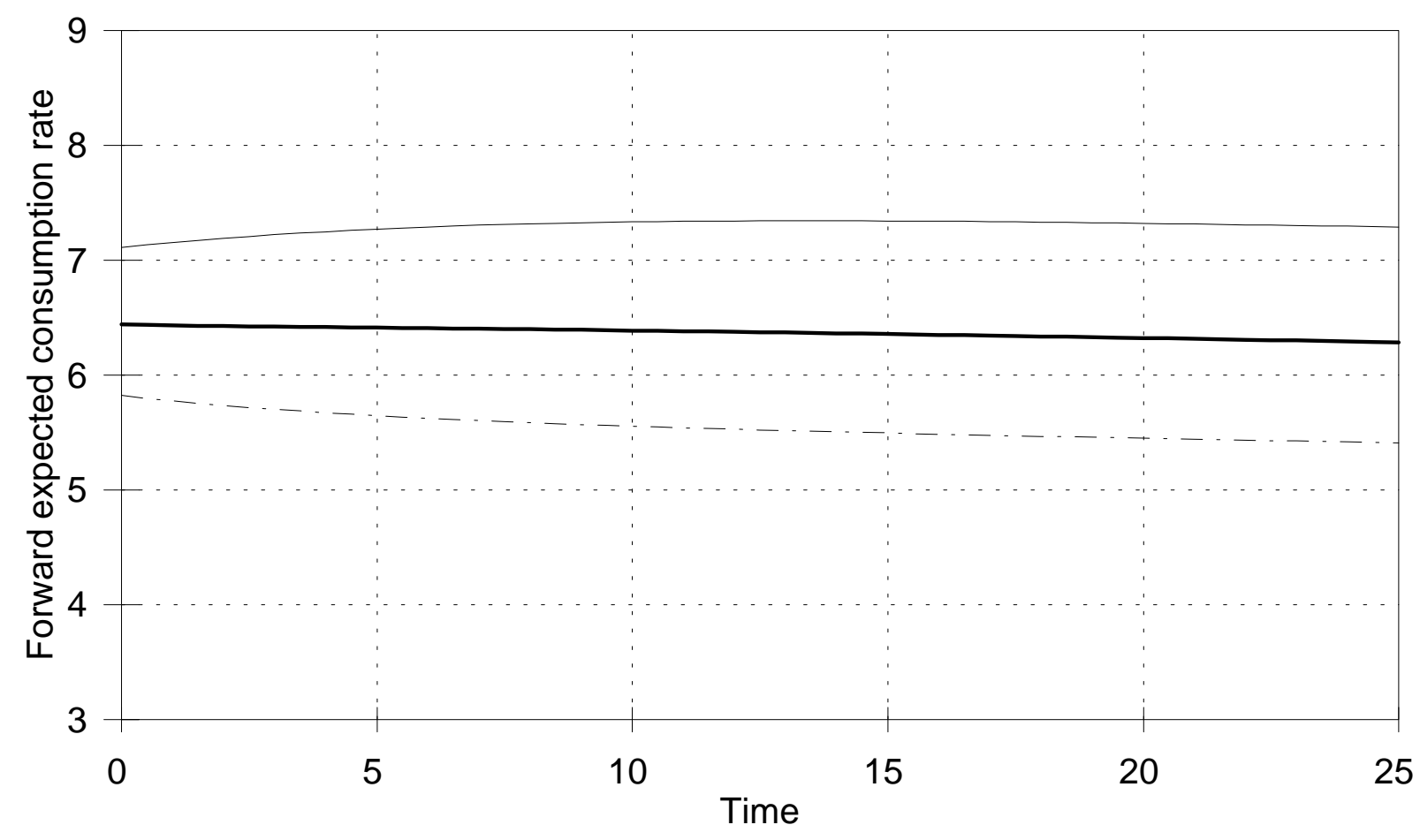

$$
--r=0.01-r=0.04-r=0.07
$$

Figure 5: Expected consumption patterns for an investor with constant relative risk aversion, $\mathbf{R R A}=\mathbf{4 . 0 0}$ and time horizon $T=\mathbf{2 5}$. The figure displays the expected consumption streams under the forward-adjusted martingale measures for the three different Vasicek term structures of forward interest rates displayed in Figure 1. 


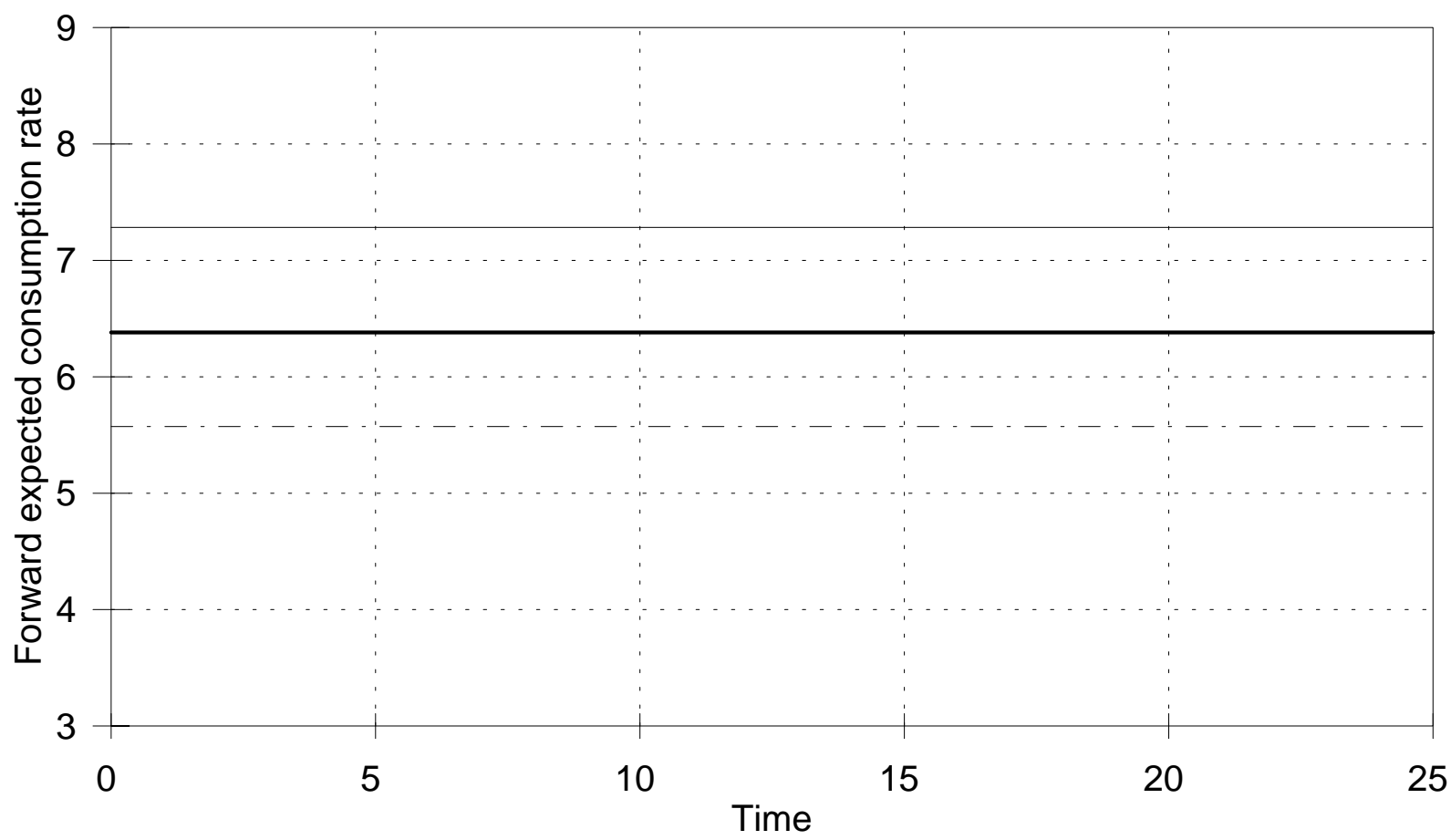

$$
--r=0.01-r=0.04-r=0.07
$$

Figure 6: Expected consumption patterns for an investor with constant relative risk aversion, $\mathbf{R R A}=\infty$ and time horizon $T=\mathbf{2 5}$. The figure displays the expected consumption streams under the forward-adjusted martingale measures for the three different Vasicek term structures of forward interest rates displayed in Figure 1. 